Cahiers $d u$ MONDE RUSSE

\section{Cahiers du monde russe}

Russie - Empire russe - Union soviétique et États indépendants

$49 / 1 \mid 2008$

Réformes et société en Asie centrale tsariste et soviétique

\title{
Lignages et pouvoirs locaux
}

L'indigénisation au kirghizstan soviétique (années 1920-1930)

Isabelle Ohayon

\section{(2) OpenEdition}

\section{Journals}

Édition électronique

URL : https://journals.openedition.org/monderusse/9123

DOI : $10.4000 /$ monderusse. 9123

ISSN : $1777-5388$

Éditeur

Éditions de l'EHESS

Édition imprimée

Date de publication : 25 février 2008

Pagination : 145-182

ISBN : 978-2-7132-2195-8

ISSN : $1252-6576$

Référence électronique

Isabelle Ohayon, «Lignages et pouvoirs locaux », Cahiers du monde russe [En ligne], 49/1 | 2008, mis en ligne le 01 janvier 2009, consulté le 03 septembre 2022. URL : http://journals.openedition.org/ monderusse/9123; DOI : https://doi.org/10.4000/monderusse.9123 
chercher : repérer : avancer

Cet article est disponible en ligne à l'adresse :

http://www.cairn.info/article.php?ID REVUE=CMR\&ID NUMPUBLIE=CMR 491\&ID ARTICLE=CMR 4910145

Lignages et pouvoirs locaux. L'indigénisation au kirghizstan soviétique (années 1920-1930)

\title{
par ISABELLE OHAYON
}

\section{| Editions de l'EHESS | Cahiers du monde russe}

\author{
2008/1 - Vol 49 \\ ISSN 1252-6576 | ISBN 9782713221958 | pages 145 à 182
}

Pour citer cet article :

—OHAYON I., Lignages et pouvoirs locaux. L'indigénisation au kirghizstan soviétique (années 1920-1930), Cahiers du monde russe 2008/1, Vol 49, p. 145-182.

Distribution électronique Cairn pour les Editions de l'EHESS.

(C) Editions de l'EHESS. Tous droits réservés pour tous pays.

La reproduction ou représentation de cet article, notamment par photocopie, n'est autorisée que dans les limites des conditions générales d'utilisation du site ou, le cas échéant, des conditions générales de la licence souscrite par votre établissement. Toute autre reproduction ou représentation, en tout ou partie, sous quelque forme et de quelque manière que ce soit, est interdite sauf accord préalable et écrit de l'éditeur, en dehors des cas prévus par la législation en vigueur en France. Il est précisé que son stockage dans une base de données est également interdit. 


\title{
LIGNAGES ET POUVOIRS LOCAUX
}

\author{
L’indigénisation au Kirghizstan soviétique \\ (années 1920-1930)
}

\begin{abstract}
«Les rapports lignagers, le combat contre les reliquats des rapports lignagers et groupusculaires demeurent des questions de première importance chez nous en Kirghizie. La lutte des lignages et des groupuscules est aujourd'hui l'arme principale de l'ennemi de classe, l'arme des bays et des manap, elle leur permet de contaminer les kolkhozes de l'intérieur ainsi que les appareils soviétiques sur place et, de cette façon, d'esquiver et de pervertir la ligne de classe. »1
\end{abstract}

Ces propos tenus en 1932 lors d'un plénum du comité régional kirghiz du parti reflètent la position du pouvoir soviétique envers les institutions lignagères dans les sociétés pastorales d'Asie centrale, tant en Kirghizie que dans les autres républiques nomades ${ }^{2}$, et sa grande méfiance vis-à-vis de toute forme de structure communautaire dont le principe d'organisation menacerait la «construction soviétique ». Au même titre que la participation aux formations dites « groupusculaires » qui obsédait les instances répressives d'URSS durant le premier stalinisme, le recours à des logiques lignagères dans la sphère politique faisaient l'objet de poursuites. Elles constituaient le leitmotiv des rapports qui rendaient compte de la difficulté de mettre en œuvre les projets de l'État, soit notamment la formation des appareils du parti et de l'administration, et la collectivisation. Cette figure récurrente du discours au Kirghizstan, moins fréquente dans les sources d'archives au Kazakhstan à la même

1. CGA PD KR, (Central’nyj gosudarstvennyj arhiv političeskoj dokumentacii Kyrgyzskoj Respubliki - Archives centrales d'État de la documentation politique de la République kirghize) 10/1/370/11b, Sténogramme du 4e Plenum du comité régional du Parti communiste kirghiz, 15/01/32, traduction de l'auteur.

2. Adrienne L. Edgar, Tribal Nation: The Making of Soviet Turkmenistan, Princeton : Princeton University Press, 2004; Isabelle Ohayon, La sédentarisation des Kazakhs dans 1'URSS de Staline, collectivisation et changement social (1928-1945), P. : Maisonneuve et Larose, 2006. 
période en dépit de l'égale importance du phénomène, avant même de renseigner l'historien sur les pratiques à l'œuvre dans la société kirghize, donne à voir la perception que les agents du pouvoir avaient de la société kirghize et singulièrement du rôle des institutions de la parenté. Le fait lignager, tel qu'il apparaît dans les archives du parti communiste kirghiz et en particulier dans les rapports de la police politique mais aussi dans les archives régionales de l'oblast' de Naryn, constitue ainsi un objet historique à double titre. Il permet d'une part de mettre en évidence le degré de connaissance que le pouvoir avait de la société « traditionnelle » kirghize et d'en analyser ses choix de gouvernement. D'autre part, il livre, dans une certaine mesure, des éléments de micro histoire sur les pratiques sociales et politiques des autochtones durant la mise en place des appareils locaux dans le contexte de l'indigénisation. Mais bien évidemment, le regard des agents de l'OGPU sur le fonctionnement de la société kirghize constitue un prisme éminemment partial, pétri des supposés et des catégories d'analyse propres à la grille de lecture bolchevique des classes sociales et des stades de développement. De surcroît, les auteurs des rapports utilisés ici, souvent d'origine européenne mais vraisemblablement secondés par des informateurs kirghiz, n'étaient pas formés pour mener des enquêtes de parenté. Le caractère parfois approximatif ou mal approprié de leurs outils descriptifs implique ainsi de rechercher derrière les notions employées la réalité ethnologique à laquelle elles renvoient. Ainsi, la difficulté de traitement de ces sources, dans lesquelles il faut cheminer par hypothèses, nous interdit malheureusement de prétendre à une anthropologie historique des usages de la parenté dans les appareils locaux ${ }^{3}$.

Pour autant, la mention répétée des problèmes liés aux lignages dans les organes de direction et d'administration nous permet de proposer une certaine histoire de l'indigénisation au niveau local, en resserrant la focale sur les comités exécutifs de région (oblast' ou kanton) et de district (rajon), sur les conseils de village (sel'sovet), et sur les comités de district du parti, afin de prendre la mesure des logiques de recrutement et des conditions de l'exercice du pouvoir. Pour ce faire, nous avons privilégié l'étude de régions particulières du Kirghizstan, caractérisées par la prédominance de l'élevage pastoral et par une composition ethnique très homogène, de façon à ne faire intervenir que marginalement la présence des Européens, cette variable changeant considérablement la configuration politique locale. La colonisation tsariste de la Kirghizie s'est en effet traduite par l'installation progressive de cantonnements cosaques puis de la paysannerie slave essentiellement dans les régions de plaine ou de piémont du nord du pays (régions de $\breve{C} u$, de Talas, de Piškek, rivage septentrional du lac Issyk-Kul') propres à la mise en valeur de la terre. Les régions de haute montagne et leurs grands bassins intramontagnards d'altitude telles que celles de Kočkor, de Naryn, du Susamyr, d'Atbaši ou encore de l'Alaj (cf. cartes p. 192 et 193) restaient très superficiellement investies par les colonies de peuplement européen, même durant la période soviétique. Pour cette

3. L'histoire orale pourrait probablement répondre à cette ambition, si tant est que la mémoire retienne jusqu'aux stratégies les plus fines déployées aux époques troubles de l'instauration du pouvoir soviétique. 
raison, nombre des documents et des cas d'études évoqués dans cet article feront référence à la région de $\mathrm{Naryn}^{4}$ et plus rarement à d'autres districts de haute montagne tels que celui d'Atbaši, par exemple.

Comme en témoignent ainsi les sources d'archives, la structuration des appareils politiques en fonction des relations de parenté dans les régions d'élevage à forte majorité kirghize était bien connue des autorités de la République autonome kirghize tout comme du pouvoir central soviétique et était pensée comme un véritable frein à la construction de l'État et de la société soviétique. À la fois instrumentalisé par les dirigeants et stigmatisé dans les discours officiels, ce phénomène s'inscrivait dans une expérience déjà longue de cooptation des élites traditionnelles par l'administration impériale russe. Conscient de cet antécédent, le pouvoir soviétique fut confronté, durant sa mise en place en Asie centrale pastorale, au défi de fonder son autorité sur une adhésion idéologique et ipso facto de rompre la logique coloniale de cooptation des chefs de lignages. D'autant plus que la doctrine bolchevique prétendait détruire l'organisation sociale lignagère qui était théorisée comme la superstructure d'une « économie nomade semi-féodale » ${ }^{5}$. En même temps, la politique d'indigénisation des cadres (korenizacija) lancée à l'échelle de l'ensemble de l'URSS au début des années 1920, rendait difficile voire impossible la neutralisation des puissantes structures lignagères qui demeuraient des institutions de référence régulant les rapports sociaux et la vie politique des Kirghiz. En effet, conformément à ses objectifs, cette politique a conduit à faire entrer la société kirghize dans les organes du jeune État soviétique, y introduisant de ce fait une culture politique et des pratiques relevant de logiques qu'elle vilipendait par ailleurs. La nouvelle composition des administrations et les caractéristiques sociologiques des recrues mettaient à l'épreuve la capacité des nouveaux organes locaux à satisfaire les exigences du pouvoir. Cette situation que nous nous attacherons à décrire ici, nous conduit ainsi à interroger les modalités de mise en place et d'exercice de l'autorité de l'État dans les appareils d'échelon inférieur, en posant notamment la question des interactions entre les critères de légitimité soviétiques et autochtones.

L'action de l'État face aux lignages et inversement la façon dont les lignages usaient du nouvel instrument politique que constituaient les récents appareils seront considérées dans une perspective dynamique qui tiendra compte de la complexité de la période examinée ici. Opérée dès les années 1923-1924 en Kirghizie, l’indigénisation était encore à l'œuvre quand débutèrent les programmes de sédentarisation et de collectivisation en 1929. La confrontation d'une logique de confiance envers la population kirghize et d'une logique de répression incarnée par la coercition collectiviste, la sédentarisation forcée et la déportation des élites traditionnelles a alors provoqué de fortes tensions. Entre 1929 et 1934, mais particulièrement entre

4. L'attention particulière portée à la région de Naryn résulte de recherches conduites entre 2005 et 2006 dans le cadre du programme ACI « nomadisme, identité, ethnicité, espaces et pouvoirs locaux (Russie, Kirghizstan, Niger, Mauritanie)» dirigé par André Bourgeot (Laboratoire d'anthropologie sociale).

5. Cf. le discours du Premier secrétaire du Parti communiste de la république autonome du Kazakhstan, in Ohayon, La sédentarisation des Kazakhs...,p. 115. 
1931 et 1934, l'hostilité générale du régime à l'égard des éleveurs kirghiz s'est également traduite par la stigmatisation et la condamnation des lignages comme acteurs politiques, la collectivisation-sédentarisation bouleversant les règles de fonctionnement local. Ainsi, entre le début des années 1920 et le milieu des années 1930, le cheminement des lignages kirghiz dans les organes politiques locaux connut différentes évolutions qui seront explorées ici. Elles nous permettront d'interroger la nature du pouvoir soviétique à cette époque et le rôle de la société kirghize comme acteur de cette histoire.

\section{1) L'indigénisation appliquée : le cas de la région de Naryn}

\section{Les cadres à l'échelon régional : un modus vivendi entre recrutement européen et kirghiz}

L'indigénisation des appareils était le mot d'ordre de la politique nationale des années 1920 et ce, jusqu'en 1932, en URSS ${ }^{6}$. Elle consistait, d'une part, à attirer les populations autochtones dans les appareils de direction afin de donner au pouvoir un visage familier, local et national et de s'assurer que les fonctionnaires locaux connaissaient la langue, le mode de vie et les mœurs de leurs administrés ${ }^{7}$. D'autre part, la politique d'indigénisation devait comprendre un volet de formation des cadres allochtones à parler les langues locales, qui était destiné non seulement à briser la distance entre le pouvoir central associé aux Russes et les représentations locales, mais aussi à renforcer un outil de communication et de propagande fondamental pour le régime. Cette initiative, l'une des plus difficiles à mettre en œuvre, rencontrait des obstacles de taille dont témoigne cet extrait du roman de l'écrivain et militant bolchevik D.A. Furmanov où il relate les activités des membres allochtones des comités du parti en Kirghizie en 1919:

Mais la masse musulmane, comment donc l'approcher quand on ne sait pas la langue locale ? Et sitôt que l'on en vint à ce point, d'elles-mêmes passèrent à l'arrière-plan les discussions sur les programmes, les paragraphes et les alinéas des statuts, les instructions, les méthodes, etc. [...] On discuta beaucoup, longtemps [...] On s'aperçut finalement que toutes nos décisions n'avaient de sens que pour quelques membres isolés, perdus dans les villages de colons et les stanicy cosaques. Au plus profond du district, nos décisions ne parviennent pas, nous le savons, le comprenons, le voyons nous-mêmes. Et, harcelés de doutes quant au sérieux de notre action, on décida assez rapidement, [...] de créer des cours de langue indigène pour les cadres qui venaient du centre. [...] Oh, je ne suis pas près d'oublier le spectacle de notre étude du kirghiz : dans une salle de classe, chacun

6. Terry Martin, The affirmative Action Empire: Nations and Nationalism in the Soviet Unions, 1923-1939, Ithaca, Londres : Cornell University Press, 2001.

7. Juliette Cadiot, Le laboratoire impérial : Russie-URSS 1860-1940, P. : CNRS Éditions, 2007, p. 135 . 
derrière son pupitre comme des écoliers, nous étions assis, nous, les chefs et dirigeants du parti : juges, responsables des services secrets, militaires et civils, jeunes et vieux [...] Au début nous étions consciencieux et appliqués jusqu'à la pédanterie. [...] La lune de miel de notre éducation indigène passa vite $[\ldots]$; force et patience nous manquaient pour consacrer notre temps à cette occupation supplémentaire, alors que nous nous hâtions d'une réunion à l'autre $[\ldots] .8$

Ainsi, la difficulté voire l'incapacité à organiser l'apprentissage du kirghiz expliqua longtemps la faiblesse des directions régionales dans les territoires majoritairement kirghiz tout comme l'indispensable présence de cadres d'origine autochtones dans les appareils. Telle qu'elle se révèle dans les nombreux rapports commandés par le Comité central de Kirghizie sur le fonctionnement des organes locaux à partir de l'année 1927 jusqu'en 1933, la situation des appareils de direction et d'administration kirghiz de la région de Naryn (kanton) et qui plus est des districts et des sel'sovety, est à cet égard parfaitement représentative.

Le kanton de Naryn, région de haute montagne située à une altitude comprise entre 2000 et 6000 mètres, était alors doté d'une population de 101000 habitants en $1928^{9}$ composée à plus de $95 \%$ de Kirghiz. La rudesse du milieu et du climat, sec, froid et continental, associée à la très faible densité de population (entre trois et quatre habitants au $\mathrm{km}^{2}$ ) en faisait une terra incognita particulièrement hostile pour les administrateurs pionniers soviétiques. La ville de Naryn, qui reçut ce statut en 1928, s'étendait le long d'une unique artère enserrée par deux flancs de montagne. Ancienne halte sur la voie caravanière vers Kašgar, en déclin depuis la fin du XVIII ${ }^{\mathrm{e}}$ siècle, elle avait été reliée par des routes carrossables à la frontière chinoise au sud, à la vallée du Ferghana à l'ouest et à la région du Semireč'e au nord par les autorités tsaristes au début du $\mathrm{XX}^{\mathrm{e}}$ siècle ${ }^{10}$. Cependant, elle restait toujours très enclavée et accessible depuis les deux grandes villes du pays, Frunze et Oř, en plus de deux jours de route. En 1928, un an après avoir été désignée chef-lieu de la région et du district éponyme, la ville comptait quelque 3000 habitants $^{11}$. Elle accueillait principalement les représentations administratives régionales et locales soviétiques, des services, trois petites fabriques et magasins d'état. Ces établissements employaient une majorité d'Européens, au parcours souvent curieux car, parfois venus de Russie occidentale ou méridionale (Nijni-Novgorod, Samara), ils avaient trouvé refuge dans ces confins soviétiques pour échapper à leur condition paysanne et à son

8. Extrait de Dmitrij A. Furmanov, Mjatež, Sobranie sočinenij [La révolte. Euvres complètes], t. 2, M. : Izd-vo hudožestvennoj literatury, 1960, cité par Guy Imart, Rémy Dor, Le chardon déchiqueté : le nomade et le commissaire (être Kirghiz au $X X^{e}$ siècle), Aix-en-Provence : Université de Provence, 1982, p. 82-83.

9. GANO (Gosudarstvennyj arhiv Narynskoj oblasti - Archives d'État de la région de Naryn), 2/1/67/7, « Doklad administrativno-organizacionnoj dejatel'nosti narynskogo kantispolkoma za period s 1 nojabrja 1927 po 15 janvarja 1928 » [Rapport d'activité administrative et organisationnelle du comité exécutif de kanton de Naryn pour la période du $1^{\mathrm{er}}$ novembre 1927 au 15 janvier 1928$]$.

10. Issyk-kul', Naryn : enciklopedija [Issykl-kul’, Naryn : encyclopédie], Frunze : KSE, 1991, p. $125-126$

11. Naryn oblusu : enciklopedija [L'oblast' de Naryn : encyclopédie], Biškek : KE, 1998, p. 50 
cortège de mesures répressives. Certains d'entre eux avaient à leur actif de multiples expériences de travail (industriel, politique, paysan, ...) dans les villes les plus diverses d'URSS ${ }^{12}$. Les Européens d'Asie centrale, relativement nombreux parmi les cadres du kanton, fuyaient eux la décosaquisation, d'autres venaient juste trouver, à Naryn, une situation. Ainsi, la physionomie générale du personnel d'encadrement européen était-elle hétéroclite. Associée aux particularités des recrues kirghizes, elle formait un singulier tableau, dans un environnement géographique et matériel pour le moins ardu. La répartition ethnique par catégorie d'employés, pour l'ensemble des institutions de création soviétique du kanton de Naryn, implantée dans la ville du même nom, répondait quant à elle aux ambitions de la politique d'indigénisation et présentait globalement les caractéristiques suivantes :

\section{Tableau $1:$ L'indigénisation dans les 20 établissements administratifs et de service du kanton de Naryn en $1928^{13}$}

\begin{tabular}{|l|c|c|c|}
\hline Catégorie d'employés & Européens & Kirghiz & Part des Kirghiz (en \%) \\
\hline Cadres dirigeants & 30 & 10 & 33,3 \\
\hline Cadres techniques & 131 & 81 & 38,4 \\
\hline Total & $\mathbf{1 6 1}$ & $\mathbf{9 1}$ & $\mathbf{3 6 , 1}$ \\
\hline Dont membres du parti & 43 & 19 & 30,6 \\
\hline
\end{tabular}

Bien que les détails de la répartition par secteurs ne soient pas ici restitués, ces données illustrent nettement la sous-représentation des Kirghiz dans les établissements de la région. En effet, la ville de Naryn qui se construisait en tant que telle à cette époque, ne comptait environ que 300 foyers européens, soit la quasi-totalité des Européens de l'ensemble de la région. Ces derniers occupaient de fait la majorité des postes politiques et techniques et la plupart des emplois du secteur des entreprises (usines, magasins) ${ }^{14}$. Il faut pourtant nuancer cette analyse et rendre compte de la spécificité des places occupées par les Kirghiz : parmi les postes dirigeants les plus importants, au Comité exécutif du kanton lui-même et au parquet (prokuratura), les Kirghiz occupaient quatre des sept postes de haute responsabilité ${ }^{15}$, ce qui souligne le

12. GANO, 73/1/98/1-5 ; GANO, 73/1/98/6-7 ; GANO, 73/1/98/11-15 ; GANO, 73/1/98/1720 ; GANO, 73/1/98/43-44 ; GANO, 73/1/98/49-50 : série d'interrogatoires des employés de l'Union de l'élevage du district (Rajživsojuz), et de l'administration du commerce (Gostorg) suite à leur dénonciation pour des petits méfaits (insultes, petits détournements), mai 1930.

13. CGA KR (Central’nyj gosudarstvennyj arhiv Kyrgyzskoj Respubliki - Archives centrales d'État de la République kirghize), 21/2/197/23, Plan raboty komissii pri CIK-e po nacionalizacii apparata s 06/02/1928 po 01/03/1928 [Plan de travail de la commission de nationalisation de l'appareil sous l'égide du Comité central du parti, du 06/02/1928 au 01/03/1928]. Document établissant la liste des administrations et leur composition nationale.

14. GANO, 2/1/38/3, Protokol $n^{\circ} 26$. Vneočerednogo zasedanija Prezidiuma narynskogo kantispolkoma, 01/07/1928 [Procès-verbal n² 26. Réunion extraordinaire du Présidium du comité exécutif de kanton de Naryn], GANO, 73/1/98/1-5, Protokol n 1 . Pervoe zasedanie po čistke sovapparata narynskogo rajživsojuza [Procès-verbal $n^{\circ} 1$. Première réunion sur la purge de l'appareil soviétique de l'union de l'élevage de Naryn], 14/05/1930.

15. CGA KR, 21/2/197/23. 
souci du système d'octroyer les postes symboliquement significatifs aux autochtones. Par ailleurs, à cette époque où la politique soviétique se nourrissait encore de l'utopie de «l'indigénisation réciproque » et était animée par des bolcheviks militants, parmi les cadres européens du kanton de Naryn, quarante disaient maîtriser la langue kirghize, quarante-deux en avoir une connaissance moyenne, lesquels représentaient environ $45 \%$ des cadres non kirghiz ${ }^{16}$. La connaissance la plus large du kirghiz, à défaut du russe qui n'était pas parlé par les autochtones, était surtout vouée à permettre la correspondance entre les échelons de rajon et de sel'sovet avec le kanton ${ }^{17}$, dans lesquels, quand les cadres étaient alphabétisés, les documents étaient prioritairement rédigés en kirghiz. En effet, les écritures produites dans les districts et les communes au cours des années 1920 et jusqu'à la deuxième moitié des années 1930, si elles existaient, prenaient la forme de notes maladroites rédigées sur des papiers de fortune, par des fonctionnaires très récemment instruits ${ }^{18}$.

La question de l'alphabétisation était précisément au cœur du problème d'indigénisation des appareils car elle conditionnait sinon le recrutement des cadres au moins l'attribution de fonctions de responsabilité. Or, la très grande majorité des Kirghiz était analphabète ; quant aux membres kirghiz du parti, ils étaient, pour la plupart, illettrés et n'avaient reçu aucune éducation scolaire. Par-delà l'alphabétisation elle-même et comme partout en URSS mais avec une acuité peut-être plus grande, le problème de la formation en général se posait, tant sur le plan technique que politique, à tel point qu'elle constituait une préoccupation omniprésente dans les rapports envoyés au centre sur le fonctionnement des administrations. Cette lacune faisait l'objet de critiques sévères et justifiait d'après les voix les plus intransigeantes, la plupart des dysfonctionnements. Parmi les cadres régionaux de Naryn pour lesquels nous disposons d'éléments biographiques, les figures suivantes illustrent parfaitement le niveau de formation des cadres à l'échelon régional :

Tableau 2 : Profil des cadres principaux du comité exécutif du kanton de Naryn ${ }^{19}$

\begin{tabular}{|c|l|l|l|l|}
\hline $\begin{array}{c}\text { Année } \\
\text { de } \\
\text { naissance }\end{array}$ & $\begin{array}{c}\text { Lieu de } \\
\text { naissance/ } \\
\text { nationalité }\end{array}$ & \multicolumn{1}{|c|}{ Formation } & \multicolumn{1}{c|}{$\begin{array}{c}\text { Passé } \\
\text { politique }\end{array}$} & $\begin{array}{l}\text { Fonction précédente/ } \\
\text { actuelle }\end{array}$ \\
\hline 1903 & $\begin{array}{l}\text { Tokmak/ } \\
\text { kirghiz }\end{array}$ & $\begin{array}{l}\text { Études secondaires, } \\
\text { instituteur, forma- } \\
\text { tion politique supé- } \\
\text { rieure à Tachkent }\end{array}$ & $\begin{array}{l}\text { Membre du } \\
\text { PCR(b) } \\
\text { depuis 1925, } \\
\text { syndiqué }\end{array}$ & $\begin{array}{l}\text { Professeur à l'institut } \\
\text { pédagogique de } \\
\text { Tachkent/ } \\
\text { Secrétaire principal }\end{array}$ \\
\hline
\end{tabular}

16. GANO, 2/1/67/27, «Doklad administrativno-organizacionnoj dejatel'nosti orgotdela narynskogo kantispolkoma za 1927/1928 » [Rapport d'activité administrative et organisationnelle du département d'organisation du comité exécutif de kanton de Naryn pour 1927/1928].

17. CGA KR, 21/2/140/22-23, Otčetnyj doklad Narynskogo Kantispolkoma Kir ASSR o dejatel'nosti za god, janvier 1928 [Exposé bilan du comité exécutif de kanton de Naryn de la république autonome de Kirghizie pour l'année].

18. Cf. dossiers GANO, 73/1/382 ; GANO, 73/1/383, GANO, 73/1/416, GANO, 73/1/465.

19. CGA KR, 21/2/190/36-37 ; CGA KR, 21/2/190/38-39, CGA KR, 21/2/190/40-41, CGA KR, 21/2/190/56 ; CGA KR, 21/2/190/57 : fiches biographiques des cadres du kantispolkom, datant de 1927. 


\begin{tabular}{|l|l|l|l|l|}
\hline $\begin{array}{c}\text { Année } \\
\text { de } \\
\text { naissance }\end{array}$ & $\begin{array}{l}\text { Lieu de } \\
\text { naissance/ } \\
\text { nationalité }\end{array}$ & \multicolumn{1}{|c|}{ Formation } & \multicolumn{1}{|c|}{$\begin{array}{c}\text { Passé } \\
\text { politique }\end{array}$} & $\begin{array}{l}\text { Fonction précédente/ } \\
\text { actuelle }\end{array}$ \\
\hline 1900 & $\begin{array}{l}\text { Naryn/ } \\
\text { kirghiz }\end{array}$ & $\begin{array}{l}\text { Éducation primaire, } \\
\text { cours de pédagogie à } \\
\text { Tachkent, fils de } \\
\text { dehkan }{ }^{20}\end{array}$ & $\begin{array}{l}\text { Membre du } \\
\text { PCR(b) } \\
\text { depuis 1924, } \\
\text { syndiqué }\end{array}$ & $\begin{array}{l}\text { Directeur nommé de } \\
\text { l'éducation populaire } \\
\text { de Naryn/Instructeur }\end{array}$ \\
\hline 1907 & $\begin{array}{l}\text { Kuban/ } \\
\text { russe }\end{array}$ & $\begin{array}{l}\text { Éducation secondaire } \\
\text { et spécialisée, fils de } \\
\text { paysan }\end{array}$ & $\begin{array}{l}\text { Membre des } \\
\text { komsomols } \\
\text { depuis 1919 }\end{array}$ & $\begin{array}{l}\text { Secrétaire de Naryn/ } \\
\text { Secrétaire technique }\end{array}$ \\
\hline 1905 & $\begin{array}{l}\text { Žitomir/ } \\
\text { juif } \\
\text { d'Ukraine }\end{array}$ & $\begin{array}{l}\text { Études secondaires, } \\
\text { faculté des } \\
\text { travailleurs, fils } \\
\text { d'instituteur }\end{array}$ & $\begin{array}{l}\text { Membre des } \\
\text { komsomols } \\
\text { depuis 1920 }\end{array}$ & $\begin{array}{l}\text { Instructeur en chef de } \\
\text { Naryn/ idem dans le } \\
\text { département d'orga- } \\
\text { nisation }\end{array}$ \\
\hline 1900 & $\begin{array}{l}\text { Kočkor/ } \\
\text { kirghiz }\end{array}$ & $\begin{array}{l}\text { Éducation primaire, } \\
\text { 3 mois de cours de } \\
\text { pédagogie, fils de } \\
\text { paysan pauvre }\end{array}$ & $\begin{array}{l}\text { Membre du } \\
\text { PCR(b) } \\
\text { depuis 1926, } \\
\text { syndiqué }\end{array}$ & Jeune instructeur \\
\hline
\end{tabular}

Toutes les catégories indiquées dans les enquêtes biographiques du parti sur les cadres ne sont pas reprises ici. Cependant, on peut d'emblée conclure à la grande jeunesse des cadres (ils ont entre 20 et 27 ans), cette génération constituant un gage de fidélité au régime, sa formation étant nécessairement assurée par les structures soviétiques. Les deux figures non kirghizes ici sont parfaitement représentatives du militant communiste bolchevik impliqué dans l'œuvre de soviétisation des « confins arriérés », qui, après une carrière parfois longue dans les organes (le juif d'Ukraine a occupé une dizaine de postes en Ukraine avant d'arriver à Naryn, dont celui de responsable d'un groupe secret de l'OGPU à la frontière soviétique occidentale) s'engage ou est forcé de s'engager loin de sa «patrie». Enfin, les Kirghiz présentent un passé militant beaucoup moins riche et plus récent, ce qui témoigne là aussi d'un phénomène courant : l'engagement tardif des autochtones dans les structures de direction de ce niveau qui nécessite une formation acquise seulement dans les années 1924-1926, laquelle était souvent assurée à Tachkent, alors centre politique pour l'Asie centrale. Par ailleurs, l'origine régionale des Kirghiz est assez diversifiée à ce niveau de direction, bien que l'on puisse considérer que le ressortissant de Kočkor soit de la grande région de Naryn. Elle résulte d'une politique délibérée d'affectation des cadres kirghiz formés autant que de la pénurie de ces derniers qu'il faut aller chercher dans toutes les régions de Kirghizie.

20. Le terme centre-asiatique dehkan renvoie au statut de paysan, sans préjuger pour autant de l'activité agricole principale, et peut désigner dans les usages administratifs tant un éleveur qu'un agriculteur. 


\section{La réalité de l'indigénisation en face : incompétence des recrues et déficience des pratiques administratives à l'échelle locale}

Le profil social des cadres d'échelon inférieur (district et sel'sovet) qui dépendent hiérarchiquement du kanton, est moins bien renseigné, en particulier pour les régions à dominante kirghize. Pour le kanton de Naryn, les données disponibles rendent compte de la présence quasi-exclusive de Kirghiz aux postes de président (predsedatel') et de vice-président (zam-predsedatel') des districts. Parmi les dixhuit personnes en exercice à ces postes dans les neufs districts du kanton, toutes sont kirghizes et membres du parti. Elles appartiennent à une génération âgée de 19 à 36 ans, qui rappelle la jeunesse des cadres de kanton. La moitié de ces dirigeants est catégorisée comme illettrée (malogramotnyj).

Au niveau encore plus bas des sel'sovety (littéralement « conseil de village » qui renvoie ici à l'administration d'un groupe d'aouls ou de kolkhozes) de Kurtka et d'Aktala, on trouve les figures de pouvoir suivantes, toutes kirghizes :

Tableau 3 : cadres des sel'sovety de Kurtka et d'Aktala au 04/11/192921

\begin{tabular}{|c|c|c|c|c|}
\hline Nom & Fonction & Age & $\begin{array}{l}\text { Extraction sociale/ } \\
\text { niveau d'instruction }\end{array}$ & Activité politique \\
\hline $\begin{array}{l}\text { Kadyr } \\
\text { Babaev }\end{array}$ & $\begin{array}{l}\text { président de } \\
\text { Kurtka }\end{array}$ & 21 ans & pauvre/illettré & $\begin{array}{l}\text { membre des } \\
\text { komsomols, } 1^{\text {re }} \text { nomina- } \\
\text { tion à ce poste }\end{array}$ \\
\hline $\begin{array}{l}\text { Marija } \\
\text { Ismailova }\end{array}$ & $\begin{array}{l}\text { vice-présidente } \\
\text { de Kurtka }\end{array}$ & 29 ans & pauvre/illettrée & $\begin{array}{l}\text { sans parti, } 1^{\text {re }} \text { nomina- } \\
\text { tion à ce poste }\end{array}$ \\
\hline $\begin{array}{l}\text { Sultanaly } \\
\text { Murataliev }\end{array}$ & $\begin{array}{l}\text { président } \\
\text { d'Aktala }\end{array}$ & 34 ans & $\begin{array}{l}\text { dehkan moyen/ } \\
\text { illettré }\end{array}$ & $\begin{array}{l}\text { membre du parti, } \\
2^{\mathrm{e}} \text { nomination à ce poste }\end{array}$ \\
\hline $\begin{array}{l}\text { Orumbazar } \\
\text { Kajpaeva }\end{array}$ & $\begin{array}{l}\text { vice-présidente } \\
\text { d'Aktala }\end{array}$ & 43 ans & dehkan/illettrée & $\begin{array}{l}\text { sans parti, } 1^{\text {re }} \text { nomina- } \\
\text { tion à ce poste }\end{array}$ \\
\hline
\end{tabular}

On notera ici la présence systématique des femmes aux postes de vice-président qui reflète la politique d'émancipation et de politisation des femmes appliquée en priorité aux plus petits échelons de pouvoir alors que ce phénomène est moins systématique à l'échelle des districts et du kanton presque exclusivement masculins. On dispose malheureusement de trop peu d'éléments biographiques pour livrer une analyse approfondie de la composition de ces appareils d'échelon inférieur. On peut néanmoins retenir le très faible niveau d'instruction de ces cadres corrélé à un tout aussi bas niveau de politisation et d'expérience, lequel laisse préjuger d'une application aléatoire des instructions du centre régional (kanton) et républicain. La seule consultation des archives régionales de Naryn donne à voir

21. CGA KR, 21/4/91/45, Spiski členov i kandidatov CIK, otvetrabotnikov okružnogo vseh kantonnyh, rajonnyh i volostnyh ispolkomov, predsedatelej s/sovetov Kir ASSR [Listes des membres et des candidats du parti du Comité central, des responsables régionaux de tous les kantony, rajony et volosti des comités exécutifs, des présidents des sel'sovety de la république autonome de Kirghizie]. 
l'indigence des documents administratifs produits jusqu'au milieu des années 1930 par les appareils de district et de sel'sovet, rédigés a minima, dans des graphies maladroites, mêlant des phrases en kirghiz et en russe, et quand c'était en kirghiz hésitant dans l'usage des alphabets arabe ou latin ${ }^{22}$. Cette forme, qui les rend difficilement exploitables, révèle l'incapacité des administrations locales à rendre compte de leur activité, donc à être contrôlées par la voie écrite par leur hiérarchie. Plus encore, les rapports sur le fonctionnement des districts du kanton de Naryn montrent à quel point la politique définie par le kanton et la république n'était pas mise en œuvre.

Dans le district d'Atbaši qui se trouvait sous la direction du kanton de Naryn, dont on peut extrapoler les caractéristiques aux districts d'élevage voisins, compte tenu de la similitude des populations et de leurs activités, l'inspection dénonçait l'inaptitude des employés de l'appareil à travailler et à prendre des initiatives. Ces derniers n'organisaient pas leurs tâches en fonction d'un plan de travail ni d'un calendrier, contrevenant en cela au principe même de la culture du travail soviétique, celui de la planification. La direction du comité exécutif de district et des sel'sovety se contentait de répartir les fournitures de bureau et de distribuer les circulaires venues des organes supérieurs, sans assumer l'administration effective de la circonscription ni donner d'instructions propres à vérifier le travail effectué dans les sel'sovety. D'autre part, les employés du district et des sel'sovety ne respectaient pas les procédures administratives de travail et notamment ne tenaient pas d'archives ou alors très incorrectement. Les sections composées de deux ou trois personnes chargées respectivement de l'agriculture, des questions financières et fiscales, des coopératives et du commerce, et de la vie quotidienne, qui formaient l'armature des appareils de district, avaient bien été formées mais ne pouvaient justifier d'aucune activité, pas même de la tenue de réunions. Il en était de même pour l'ensemble des fonctions prévues pour une administration de district, si bien que l'appareil fonctionnait véritablement comme une coquille vide, laissant indifférente la population comme les militants locaux du parti23.

Le district d'Atbaši comme la plupart des districts de haute montagne de peuplement kirghiz, souffrait de surcroît de la topographie des lieux et de la faiblesse des moyens de communication qui ralentissaient en effet considérablement l'activité des appareils. La distance entre les lieux d'habitation et les sel'sovety allait jusqu'à $50-60 \mathrm{~km}$ et était en moyenne de 20-25 km, ce qui rendait difficiles la connaissance et l'information de la population et son accès aux prestations. Par ailleurs, les moyens en hommes ne permettaient pas de compenser ces contraintes : le district d'Atbaši ne possédait par exemple que trois miliciens à cheval qui ne se déplaçaient que si une affaire était portée à leur connaissance. Il n'existait pas de maison d'arrêt. Les services civils d'enregistrement des mariages, des décès et des

22. Cf. dossiers GANO, 73/1/382 ; GANO, 73/1/383, GANO, 73/1/416, GANO, 73/1/465.

23. CGA KR, 21/4/117/19-45, Doklad o sostojanii raboty Sovetskogo apparata v Atbašinkoj volosti Narynskogo kantona, oct-nov 1929 [Exposé sur la situation du travail dans l'appareil soviétique du volost' d'Atbaši dans le kanton de Naryn]. 
naissances, qui manquaient de formulaires et de registres, ne diffusaient pas d'information auprès de la population si bien que les actes étaient enregistrés de façon aléatoire et très partielle 24 . Ce tableau succinct de l'année 1929 sur le fonctionnement général d'une administration de district interroge directement les effets de l'indigénisation et plus généralement les conditions de la mise en place du pouvoir soviétique au niveau local. Les caractéristiques sociologiques des recrues autochtones, le manque d'encadrement, les pénuries diverses, la nature de la société à laquelle devaient s'adapter les organes soviétiques, constituaient autant d'obstacles à la satisfaction des exigences bureaucratiques du nouveau pouvoir et, par conséquent, à la construction politique soviétique. Ces contraintes, posant de facto la question du caractère inopérant du projet d'indigénisation, s'ajoutaient aux spécificités de la culture politique des lignages.

La société kirghize est en effet structurée en groupes généalogiques aux attributions variables en fonction du degré de parenté auquel ils renvoient. Cette organisation sociale peut être décrite selon le schéma idéal-typique suivant. Ce qui est traduit par plemja dans la tradition ethnographique russe désigne le segment de parenté le plus large et correspond à ce que l'on préfère appeler ici « ligne de descendance » ou « ligne généalogique » plutôt que tribu ${ }^{25}$. Celles-ci, désignées par uruu en kirghiz, qui se réfèrent à un ancêtre commun très éloigné ou parfois fictif, sont au nombre de quarante ${ }^{26}$, et sont reconnues et fixées grâce aux généalogies dont la connaissance par les Kirghiz conditionne leur appartenance au groupe. Vient ensuite l'uruk, que l'on peut traduire par lignage, qui rassemble des groupes liés par des ancêtres communs à cinq-sept générations, puis enfin le bir atanyn baldary, littéralement « enfants d'un même père », groupe de filiation, qui se définit par rapport au dernier ancêtre décédé et regroupe le plus souvent entre trois et cinq générations ${ }^{27}$. En fonction de leur taille, l'uruk et le bir atanyn baldary (rendu indifféremment par le terme rod en russe) rassemblent des communautés où toutes les personnes se connaissent et sont liées par une prescription de solidarité imposée par la filiation patrilinéaire. Fondée sur des interactions réelles et interpersonnelles, cette solidarité ne tolère pas les infractions aux règles d'entraide, qui sont sanctionnées par l'exclusion du groupe, non plus que la vengeance réglée. Elle se différencie de l'autre type de

\section{CGA KR, 21/4/117/19-45.}

25. Nous empruntons cet usage ainsi que la majorité des données suivantes sur le fonctionnement du système de parenté kirghiz à l'intervention de Svetlana Jacquesson présentée à Timia (Niger), le 28/010/2006 dans le cadre du programme ACI «nomadisme, identité, ethnicité, espaces et pouvoirs locaux (Russie, Kirghizstan, Niger, Mauritanie) » et à ses différents travaux à paraître, «Power Contest among the Kyrgyz: State versus Descent » in Roberte Hamayon, éd., Représenter le pouvoir en Asie : légitimer, consacrer, contester, « Régimes politiques et pratiques funéraires dans le Kyrgyzstan du nord», Numéro spécial d'Études mongoles, sibériennes, centre-asiatiques et tibétaines.

26. Elles sont décrites et cartographiées par Saul M. Abramzon dans Narody Srednej Azii i Kazahstana [Peuples d'Asie centrale et du Kazakhstan], M. : Izdatel'stvo Akademii nauk SSSR, 1963,p. 176.

27. Činara R. Israilova-Har'ehuzen, Tradicionnoe obščestvo kyrgyzov v period russkoj kolonizacii vo pervoj polovine $X I X-X X$ v. i sistema ih rodstva [La société traditionnelle des Kirghiz durant la colonisation russe et leur système de parenté], Biškek : Ilim, 1999, p. 130. 
solidarité plus aléatoire qui structure les relations entre uruu. En effet, celle-ci ne repose pas sur la parenté réelle mais sur l'idéologie de la parenté et mobilise des groupes qui ne se connaissent pas nécessairement. Avant tout symbolique et légitimée par les grandes généalogies consignées, elle est notamment actualisée lors des rites funéraires ${ }^{28}$. La nature à la fois idéologique et rituelle des relations entre uruu fait que les interactions réelles qu'elles engendrent peuvent changer d'une époque à l'autre, produire des alliances comme des rivalités, et explique qu'elles puissent être politiquement manipulées.

\section{2) Lignages et indigénisation : l'enracinement des logiques locales dans l'appareil soviétique}

L'administration soviétique, parce qu'elle était assez fortement indigénisée et parce qu'elle consacrait une grande partie de son activité à la récolte d'informations sur la société, maîtrisait, au moins localement, la répartition des lignes de descendances et des lignages dans les territoires administrés, comme en témoignent de nombreux documents tel un rapport de 1928 sur le kanton de Naryn ${ }^{29}$, qui énumère les lignages et identifie leur localisation. Dans certains cas, les représentants de la police politique relevaient même le ou les schémas de parenté correspondant au peuplement d'un sel'sovet ou d'un pâturage d'hiver auquel était ainsi associé un lignage complet dans la complexité de sa structure et de ses relations avec d'autres lignages $^{30}$ (cf. schéma de parenté, p. 00).

Cette abondante littérature des rapports de la police politique adressés au Comité central du parti de la république autonome de Kirghizie comportait de nombreuses critiques portant sur la collusion entre les lignages et les appareils. Parmi les griefs formulés, certains portaient sur la constitution des sel'sovety sur une base lignagère, ce qui «compliquait le travail des organes soviétiques ${ }^{31}$. Associé à d'autres critères, l'homogénéité lignagère des appareils de volost'et de sel'sovet était tenue responsable de la faiblesse de l'activité, du manque d'initiative et de planification constaté, des fraudes lors des élections notamment ${ }^{32}$. Déjà en 1933, alors que la collectivisation avait conduit à l'émigration vers la Chine des éleveurs refusant la mise en commun et la livraison obligatoire du bétail et que les appareils avaient été maintes fois purgés à la demande du centre, les rapports

28. S. Jacquesson, « Régimes politiques et pratiques funéraires dans le Kyrgyzstan du nord », Études mongoles, sibériennes, centre-asiatiques et tibétaines, à paraître.

29. GANO, 2/1/67/21-27, 13/10/1928.

30. CGA PD KR , 10/1/144/182, «Podvetv’ Džakit-S[...] Alagus 70 hoz. Kštau bulak. Genetičeskaja shema roda Mongoldor », 20/11/1927 [La sous-branche Džakit-S[...] Alagus de 70 foyers, pâturage d'hiver bulak. Schéma génétique de la tribu Mongoldor] et CGA PD KR, 10/1/144/193, «Genetičeskaja shema roda Sarybagiš kolena manap vetvi esengul » 20/11/ 1927 [Schéma génétique de la tribu Sarybagiš, branche manap, sous-branche esengul].

31. CGA KR, 21/4/117/12-45.

32. CGA KR, 21/4/117/12-45. 
adressés par la représentation de la République kirghize à Moscou au département des nationalités d'URSS, soulignaient qu'en particulier dans les districts de Naryn et d'Atbaši, les chefs politiques restaient liés à leur lignage ${ }^{33}$ et à leur ligne de descendance et que cette appartenance influençait leurs actions.

Ce constat que les différentes hiérarchies républicaine et fédérale pouvaient tirer des documents qu'elles recevaient, appelle plusieurs questions sur la nature du pouvoir dans les régions pastorales kirghizes dans les années 1920 et 1930. Elle interroge, à l'intérieur des organes politiques d'échelon inférieur, l'interaction des critères de légitimité soviétiques et autochtones et la capacité de la société kirghize à ajuster les principes d'autorité habituellement en usage. La variété des combinaisons des formes de légitimité, des formes de loyauté et des logiques politiques contradictoires exige de montrer comment le système de parenté et plus largement de relations organisait de facto les appareils soviétiques, puis comment les lignages ont pu constituer des instruments de la politique soviétique, et enfin comment au contraire ils subvertissaient à différents titres les projets de l'État.

\section{Les Kirghiz dans l'appareil : le tropisme lignager}

Comment la logique lignagère structurait-elle les appareils locaux ? Le mode de recrutement des cadres des administrations soviétiques locales dans les régions ethniquement les plus homogènes obéissait à des règles changeantes dans la mesure où la pénurie de personnes lettrées et politiquement formées parmi les Kirghiz limitait fortement les marges de manœuvre. Le critère qui intervenait pour accorder des responsabilités à un cadre de rajon était notamment le service dans l'Armée rouge, qui constituait l'une des seules garanties de la loyauté au régime, avec les expériences de formation dans un institut du parti d'Asie centrale, ce qui était beaucoup plus rare à la fin des années 1920. Le recrutement, qui était le plus souvent le résultat de pratiques de cooptation ou de parachutage, obéissait à un autre principe de base qui consistait à écarter des appareils les personnes qui avaient déjà occupé une fonction sous l'Empire et qui appartenait aux catégories sociales dites « ennemies ». Mais ces critères élémentaires étaient, dans les faits, impossibles à contrôler : durant les années 1920 et la première moitié des années 1930, on trouvait dans les appareils administratifs indigénisés un certain nombre d'anciens fonctionnaires de volost' qui devaient leur promotion à leur statut de chef de lignage, de riches propriétaires de bétail, ou tout simplement aux qualités qui résultaient de leur expérience.

Que les recrues des appareils locaux aient été des hommes de confiance de par leur parcours depuis la révolution et leur extraction sociale ou qu'elles se soient situées dans la continuité des formes d'autorité politique prérévolutionnaires, elles étaient nécessairement appréhendées selon des critères lignagers par leur entourage

33. CGA KR, 1246/1/631/1-34, «Dokladnaja zapiska Muhadži i Nazaryvskogo v svjazi s proverkoj raboty po osedaniju v KirASSR » [Note bilan de Muhadži et Nazaryvskij concernant le travail de contrôle sur la sédentarisation en république autonome de Kirghizie], fin 1933. 
politique immédiat, du fait même du primat du système de parenté kirghiz sur toute autre forme d'organisation. Quiconque occupait une fonction dans les appareils ruraux en Kirghizie était directement associé au réseau de relations auquel il appartenait. Ses choix politiques étaient compris comme ceux d'un groupe. Si bien que, par la seule présence d'autochtones dans les appareils, les rapports politiques pouvaient être lus comme des rapports de clans au sens large du terme. La constitution des appareils résultait ainsi de luttes d'intérêt qui engageaient des groupes lignagers investis dans le jeu politique local. Cette réalité était connue, comme nous l'avons vu, des dirigeants des républiques du Kazakhstan et du Kirghizstan, et, sa prise en compte intervenait de facto amplement dans les stratégies de construction locale des pouvoirs élaborées au niveau républicain.

Comme l'illustre un rapport de la police politique relatif au district de Ton, rajon situé sur la rive méridionale du lac Issyk-Kul' au sud-est de la ville de Balykčy en date du 18 février $1932^{34}$, un groupe de parenté et un appareil local pouvait intégralement coïncider. En effet, ce rapport décrit et dénonce les liens de parenté de divers degrés qui unissent les membres de l'appareil du parti et des administrationsclés du district. Il met en cause le secrétaire du Comité du parti, Kurmanaliev, en distinguant, comme rapporté ci-dessous, trois types de degrés de parenté en jeu dans le choix des cadres de direction du district : un premier degré renvoyant à la famille très proche, un degré désigné par le terme russe de rodstvennik qui correspond vraisemblablement à la famille élargie au sens de la catégorie de bir atanyn baldary et enfin un degré renvoyant au lignage uruk, ici Aryk-tukum.

Ce document, de façon peut-être plus manifeste encore que d'autres, révèle à quel point la connaissance des rapports de parenté était importante pour les pouvoirs soviétiques au Kirghizstan. Tout en montrant notamment la difficulté pour les informateurs de la police politique à maîtriser les catégories de la parenté kirghize, il affiche un talent certain à identifier les relations et les réseaux, lesquelles constituaient les enjeux principaux de la surveillance en Kirghizie dans les années 1920 et 1930. Cette description est suivie d'une analyse des conséquences de cette configuration des pouvoirs pour la population du district. D'après les auteurs du rapport, le secrétaire du comité du parti du district de Ton, Kurmanaliev, cherchait à concentrer les ressources politiques entre ses mains et à les déployer en faveur de ses seuls alliés. En réponse à la stratégie monopolistique de Kurmanaliev, les sel'sovety du district qui en ont eu les moyens se sont organisés selon les mêmes critères, afin de lutter sur des bases identiques contre les intentions de Kurmanaliev qui, du fait de sa fonction, contrôlait de nombreuses ressources politiques et matérielles. Les lignages qui n'ont pas réussi à prendre un appareil se sont tous ralliés au chef de la police politique du district dans le but d'assurer leur protection. Ainsi trois parties ont émergé : le «clan » de Kurmanaliev, les petits groupes des sel'sovety, et les mécontents aux côtés de l'OGPU. Or, c'est visiblement le clan de Kurmanaliev qui bénéficiait de l'autorité la plus grande, en particulier contre le clan de l'OGPU, notamment parce qu'il fondait ses arguments 
Tableau 4 : Les relations de parenté dans le district de Ton

\begin{tabular}{|c|c|c|}
\hline Nom de famille & Lieu de travail ou fonction & $\begin{array}{c}\text { Degré de parenté } \\
\text { avec Kurmanaliev, } \\
\text { secrétaire du comité } \\
\text { du parti du district de Ton }\end{array}$ \\
\hline 1-Sadykov & $\begin{array}{l}\text { Département de l'agitation de masse } \\
\text { du comité du parti du district }\end{array}$ & Gendre de Kurmanaliev \\
\hline 2-Sakeeva & $\begin{array}{l}\text { Secteur « femmes » du comité du parti } \\
\text { du district }\end{array}$ & $\begin{array}{l}\text { Épouse de Sadykov, sœur de } \\
\text { Kurmanaliev }\end{array}$ \\
\hline 3- Ishakov & Président du rajkolhozsojuz ${ }^{35}$ & Beau-frère de Kurmanaliev \\
\hline 4- Bakaev & $\begin{array}{l}\text { Vice-directeur du bureau des commu- } \\
\text { nications et transports }\end{array}$ & Parent (rodstvennik) \\
\hline 5- Hamekov & Vice-directeur du « port » & Parent \\
\hline 6- Khamzamulin & $\begin{array}{l}\text { Vice-président du comité exécutif de } \\
\text { district }\end{array}$ & Parent \\
\hline 7- Bajgaziev & $\begin{array}{l}\text { Vice-président du comité du parti de } \\
\text { district }\end{array}$ & Parent \\
\hline 8- Adylov & Procureur & Parent \\
\hline 9- Toktobekov & Secrétaire du comité régional du parti & Parent \\
\hline 10- Kadyrbaev & Animateur de sovkhoze & Parent \\
\hline 11-Sariev & Économiste du rajkolhozsojuz & Parent \\
\hline 12- Bolčumbaev & Président de l'union des chasseurs & Parent \\
\hline 13- Betikanov & Président du rajpotrebsojuz ${ }^{36}$ & $\begin{array}{l}\text { Du même lignage (odnogo } \\
\text { roda) « Aryk-tukum » }\end{array}$ \\
\hline 14- Atankulov & Inspecteur au département de la santé & $\begin{array}{l}\text { Du même lignage } \\
\text { «Aryk-tukum » }\end{array}$ \\
\hline 15- Džamankulov & $\begin{array}{l}\text { Directeur d'un département du } \\
\text { Kirpotrebsojuz }{ }^{37}\end{array}$ & $\begin{array}{l}\text { Du même lignage } \\
\text { «Aryk-tukum » }\end{array}$ \\
\hline 16- Abdyldaev & $\begin{array}{l}\text { Inspecteur de section de district de } \\
\text { l'Armée rouge }\end{array}$ & $\begin{array}{l}\text { Du même lignage } \\
\text { «Aryk-tukum » }\end{array}$ \\
\hline 17- Dušembiev & $\begin{array}{l}\text { Inspecteur de section de district de } \\
\text { l'Armée rouge }\end{array}$ & $\begin{array}{l}\text { Du même lignage } \\
\text { «Aryk-tukum » }\end{array}$ \\
\hline $18-[\ldots]$ & Directeur du bureau des plaintes & $\begin{array}{l}\text { Du même lignage } \\
\text { «Aryk-tukum » }\end{array}$ \\
\hline
\end{tabular}

35. Organisation de district pour la mise en place des kolkhozes.

36. Organisation de district chargée de la consommation et des biens de consommation (distribution).

37. Idem mais au niveau républicain. 
d'opposition à la police politique sur le fait qu'il défendrait les Kirghiz contre l'expropriation et en particulier les bays ${ }^{38}$ (riches éleveurs) et les manap (figures d'autorité traditionnelles) contre la déportation en Ukraine ${ }^{39}$, laquelle avait effectivement lieu dans ces années-là (cf. infra). Ces promesses se sont concrétisées par des manœuvres consistant à cacher les familles exposées et par des assassinats des membres de la police politique. D'autre part, cette situation mettait également en péril l'organisation des travaux agricoles prévus par le programme de collectivisation qui avaient pris du retard ou qui étaient inégalement répartis entre les sel'sovety et les familles du district. Ainsi, le rapport se terminait par une demande d'intervention urgente de la part des autorités républicaines.

\section{Kydyk contre Sayak, l'appareil au service des luttes claniques?}

Un tel phénomène se révèle de façon plus précise dans une autre série de rapports de l'OGPU kirghiz ${ }^{40}$ qui a le mérite de présenter un tableau très représentatif du fonctionnement politique des rajony pour le Kirghizstan. Il concerne le rajon de Balykči en 1931-1932, qui est un gros district incluant la rive occidentale du lac Issyk-Kul’ . Le territoire que recouvre cette région administrative est traditionnellement dominé par la grande tribu kirghize des Sayak, puissante par sa notoriété, son nombre et par l'espace qu'elle occupe. À la fin des années 1920 et au début des années 1930, ce district était ainsi dirigé par des Sayak : le secrétaire du comité de parti du rajon (Rajkom), les dirigeants des organes de justice (prokuratura), le président du comité exécutif de rajon (rajispolkom) et la majorité des cadres dirigeants des appareils appartenaient à cette ligne généalogique.

D'après les informateurs de la police politique, la domination des Sayak fut remise en cause lors d'un conflit qui opposa les principaux appareils locaux à un homme identifié comme appartenant au lignage Kydyk, lequel était vraisemblablement d'une autre ligne de descendance que celle des Sayak et d'une moindre profondeur généalogique, mais les rapports ne le précisent pas. Cet homme, Kodžemkulov, présidait un sel'sovet proche de la frontière chinoise et travaillait comme intermédiaire kirghiz auprès de la Komandatura de l'OGPU, chargée de garder la frontière avec la Chine et d'enrayer la contrebande, et dont le personnel était majoritairement européen. En raison de sa fonction même et de l'obstacle qu'il représentait pour les trafics en tout genre qui animaient cette frontière et qui impliquaient en particulier les riches éleveurs, Kodžemkulov faisait l'objet de poursuites de la part des organes de justice du rajon sous de faux chefs d'accusation. En réalité, et c'était exprimé ouvertement dans la presse locale, cet homme était accusé « d'être

38. Le terme bay a été transcrit ici avec un y et non un $j$ comme l'aurait voulu une translittération rigoureuse, afin qu'il reste identifiable comme relevant de la terminologie turcique des titres et fonctions, à l'image de bey, beg, etc.

39. CGA PD KR, 10/1/434/77, Lettre du délégué de la GPU du rajon de Balykči au Secrétaire du comité régional kirghiz du parti, Isakeev, 15/02/32.

40. CGA PD KR, 10/1/434/96-97, Note de l'OGPU, 26/03/32. 
un larbin des Russes (prihvostnik) et d'empêcher ses frères kirghiz d'effectuer leur commerce ». Il avait en effet dénoncé nommément les agissements des contrebandiers à une conférence régionale du parti et s'était attiré les foudres de l'ensemble des représentants politiques sayak. À tel point que les membres kydyk de son lignage, qui pourtant condamnaient aussi sa collaboration avec l'OGPU, se sont ralliés à lui pour défendre les places des Kydyk dans les appareils. Face à la menace, les Sayak ont usé de tous les moyens légaux dont ils disposaient grâce à leurs fonctions dans les organes pour neutraliser Kodžemkulov, allant jusqu'à demander au Comité du parti de la République kirghize de le démettre de ses fonctions de président de sel'sovet au motif qu'il était corrompu et dirigeait un groupuscule.

L'affaire a pris une telle ampleur que la plupart des lignages et lignes de descendance (le rapport confond les deux degrés et dénomme les deux rod en russe) présents dans le rajon de Balykči ont pris partie pour l'un ou l'autre camp. Les groupes de parenté mineurs ont profité de la situation pour dénoncer le projet des Sayak de placer uniquement des gens de leur ligne de descendance aux postes du rajon, à une période où les prochaines élections au rajispolkom agitaient la population. Confrontés à cette situation, dans le contexte de la collectivisation, à une saison où les semailles approchaient, et au risque d'assister à une guerre « clanique » dont les armes auraient été d'un côté les moyens de répression étatiques, de l'autre des stratégies de défiance aux ordres et notamment à ceux d'ensemencer, les autorités de la république (l'OGPU et le Comité du parti) décidèrent de démettre les deux leaders claniques de leurs fonctions, le Kydyk et le Sayak, et de promouvoir au poste de président du rajispolkom le représentant d'un lignage étranger à ces querelles. Mais, en aucun cas, les deux leaders ne furent sanctionnés, ils furent momentanément neutralisés. Ce que l'on peut interpréter ici comme une politique d'équilibre vis-à-vis des groupes généalogiques rappelle la logique, décrite par Adrienne Edgar ${ }^{41}$, qui a présidé à l'usage politique des lignages à la même époque dans la république soviétique du Turkménistan, bien qu'elle diffère radicalement quant à l'échelle d'intervention.

Qu'il s'agisse des Kydyk ou des Sayak, les représentants des lignages qui s'affrontaient étaient parfaitement intégrés dans les appareils et les utilisaient pour mener leur combat. Alors qu'ils ne relèvent pas de la même catégorie de groupes de parenté, puisque le premier renvoie à un uruk ou à un bir atanyn baldary et le second à une grande ligne généalogique impliquant une population plus nombreuse, la mobilisation fondée sur l'appartenance lignagère joue néanmoins un rôle prépondérant dans la lutte politique, l'identification à un groupe se faisant en dépit de ce déséquilibre. La question est pourtant de savoir ce qui relève de la solidarité de lignages et de ce qui tient de l'instrumentalisation du lignage. En effet, les Sayak utilisent le discours de la «nationalité » pour dénoncer leur ennemi tandis que les Kydyk leur opposent le discours de la légalité pour les incriminer. Ce n'est donc pas par l'invocation de l'appartenance clanique que se joue la rivalité. Pourtant, le

41. A. Edgar, « Genealogy, Class, and "Tribal Policy” in Soviet Turkmenistan, 1924-1934», Slavic Review, 60, 2 (Summer, 2001), p. 266-288 
résultat de la querelle montre que ce sont des mécanismes de solidarité lignagère qui ont fait tourner un conflit politique interpersonnel en conflit de clan, et c'est ainsi que le conflit est décrit par l'OGPU, tendant à prouver que la solidarité lignagère prime sur les intérêts particuliers: les Kydyk se rassemblent alors qu'ils n'approuvent pas la collaboration de leur homme avec l'OGPU. Un tel schéma de rivalités entre appareils associés à des lignages peut fonctionner car les appareils fondent en partie leur autorité sur leur cohésion lignagère. Le pouvoir républicain est parfaitement au fait de cette structuration mais n'intervient que quand les priorités politiques sont mises en péril par les luttes claniques, en s'imposant comme juge et arbitre.

\section{La loyauté dans le contexte de la répression : la manipulation des lignages}

Dans ces conditions, comment des formes de loyauté au régime soviétique pouvaient-elles exister? Une telle structure du pouvoir pouvait-elle satisfaire les objectifs de la politique soviétique ? Plusieurs situations nous laissent penser que la structure lignagère était exploitée ou manipulée pour appliquer des projets venus d'en haut. Une cellule du parti ou une administration qui recoupait un lignage, pouvait mettre cette coïncidence au service de l'État, si bien que le lignage, institutionnalisé dans un appareil, utilisait ses prérogatives traditionnelles pour appliquer la politique. On peut même en conclure, à certains égards, que c'est grâce à l'investissement par les lignages des appareils que la politique soviétique pouvait être appliquée, du moins dans les milieux ethniquement homogènes.

Pour autant, sur la répression proprement dite, on manque d'exemples précis pour la Kirghizie, alors qu'ils abondent pour le Kazakhstan. À plusieurs reprises, notamment pendant les épisodes répressifs de la fin des années 1920 et du début des années 1930, les rapports entre lignages ont été exploités au service de la répression au Kazakhstan. Par exemple, pendant la campagne dite « de confiscation des biens des riches propriétaires de bétail, des semi-féodaux et de leur déportation » de l'année 1928, les cellules locales du parti (à l'échelon du sel'sovet) ont instrumentalisé les inimitiés lignagères ${ }^{42}$. Il s'agissait de susciter la délation par la population kazakhe des riches éleveurs et des élites traditionnelles et, à partir des dénonciations, d'établir les listes de personnes soumises à la confiscation. En théorie, le critère de classe devait être au fondement de cette entreprise, les dominés devant dénoncer les dominants. Or les cellules locales du parti, qui étaient parfaitement conscientes de la puissance des solidarités lignagères face aux « antagonismes de classe », ont adopté une stratégie particulière. Elles ont mobilisé le savoir que la population avait sur des lignages rivaux et leurs élites politiques et économiques pour constituer les listes de personnes à confisquer. Autrement dit, les représentants du pouvoir soviétique au niveau des rajony et des sel'sovety, autochtones dans leur grande majorité et souvent dans leur intégralité, se sont appuyés sur les lignages

42. Cf. chapitre 2 in Ohayon, La sédentarisation..., p. 71-105. 
pour mettre en œuvre la politique insufflée depuis la république, en mettant à profit leur propre connaissance de la société kazakhe. Cette méthode qui consistait à instrumentaliser des lignes de fractures pertinentes pour les sociétés locales apparaissait en effet dans les directives officielles qui émanaient de la représentation républicaine du parti du Kazakhstan à destination des cellules locales :

[...] Les discussions sur les listes de bays doivent prendre en compte toutes les façons possibles d'émettre un avis pour les masses de l'aoul, en brisant les barrières de la parenté, en vérifiant les listes lors des réunions avec les habitants des aouls les plus petits, [...] en allant s'il le faut jusqu'au travail individuel avec chacun [...]. Le bétail confisqué et les autres moyens de production [doivent] être obligatoirement répartis entre les pauvres, en excluant du partage la lignée à laquelle le bay confisqué appartient..$^{43}$

Les lignages marquaient ainsi concrètement les limites d'une politique uniquement fondée sur des catégories de classes. Mais les acteurs de cette politique appartenaient eux-mêmes à un groupe de filiation, si bien que les commissions de confiscation qui étaient chargées de mettre cette politique en application, pouvaient là encore être utilisées pour satisfaire des règlements de compte personnels ou collectifs motivés par des considérations d'appartenance généalogique. Parmi les documents conservés dans le fonds de la Commission de confiscation ${ }^{44}$, on rencontre plusieurs témoignages sur l'instrumentalisation de la parenté pour confisquer et neutraliser un ennemi et, par-là, conserver le pouvoir ou y accéder. Ces témoignages, exclusivement issus d'un corpus de lettres de plaintes, attestent avec éloquence de la conjugaison de l'autorité lignagère traditionnelle et du jeune pouvoir soviétique, ainsi que de l'utilisation réciproque des prérogatives respectives de l'un et de l'autre. Une plainte du 4 janvier 1929 adressée au Sovnarkom de la RSSA kazakhe, formulée par Bošaj Mojnakov, originaire de l'okrug du Syr-Daria et déporté dans l'okrug d'Ural'sk ${ }^{45}$, faisait état d'une manipulation contre lui sur des critères lignagers. Il dénonçait sa condamnation en tant que bay à la saisie de ses biens et à sa déportation alors qu'il ne possédait pas assez de bétail pour être incriminé. Il accusait l'un de ses

43. CGA RK (Central’nyj Gosudarstvennyj Arhiv Respubliki Kazahstan - Archives centrales d'État de la répubique du Kazakhstan), 135/1/1/92-103, « Lettre ouverte à toutes les cellules du VKP(b) et tous les communistes des aouls ». Document non daté, vraisemblablement du mois de septembre ou d'octobre 1928.

44. CGA RK, fonds 135 de la «Commission de confiscation des biens des bays », organe circonstanciel créé le 27 août 1928 et dissout au printemps 1929, comprenant les procèsverbaux des réunions de la commission et de ses représentations régionales (okrug) et locales (rajon), les plans de travail de cet organe, sa correspondance avec les délégations locales, les dossiers individuels des personnes soumises à la confiscation qui se composent des attestations de confiscation, des éventuelles lettres de plainte et demandes de recours des victimes, de rapports sur l'application des condamnations et notamment de la déportation. On y trouve notamment des lettres manuscrites relevant du corpus des plaintes et des témoignages en faveur d'un condamné, rédigées le plus souvent en russe par des fonctionnaires-traducteurs et signées de la main malhabile des plaignants souvent illettrés ou analphabètes, en kazakh et en caractères arabes.

45. CGA RK, 135/1/12/68-70. 
ennemis qui, jouissant de son statut de délégué au sein de la Commission de confiscation, en aurait profité pour l'éliminer. B. Mojnakov, expliquait être victime de l'insoumission de son lignage, Žaqsybaj, à celui d'un certain Manabaj Mankiev, fils du chef du lignage Bagul, qui faisait partie des quinze délégués de la commission, au demeurant tous Bagul. Il ajoutait qu'à l'époque tsariste, il avait déjà été l'objet d'une machination similaire qui avait causé sa déportation pour « incivisme politique », alors que M. Mankiev occupait un poste d'élu local. Dans cet aoul n 9 du volost' d'Ički-Talas de la région de Taraz, la population était effectivement composée de 27 foyers du lignage Žaqsybaj et de 460 foyers appartenant à la branche des Bagul, rapport qui était attesté par le bureau des statistiques du volost'. Cette prédominance numérique d'un groupe de filiation sur l'autre se traduisait dans les appareils qui avaient toujours été dominés par les Bagul, à l'époque impériale comme soviétique. Voulant compenser ce déséquilibre, B. Mojnakov avait tenté d'imposer son autorité et d'intégrer la Commission de confiscation. Son ennemi s'était alors empressé de l'inscrire sur la liste des bays et d'exiger son arrestation par les détachements armés du chef-lieu de rajon. Là-bas, on lui refusa ce service, ce qui le conduisit à acheter, par le don d'un cheval, les faveurs du chef de la milice locale. Celui-ci vint ainsi procéder, en compagnie du représentant de la Commission de confiscation de l'aoul, à la réquisition des biens de $\mathrm{B}$. Mojnakov, à son arrestation et à sa conduite à la ville, d'où il fut déporté. L'absence d'exemples concrets pour la Kirghizie sur la combinaison des outils des campagnes de répression et des prérogatives des lignages ne nous autorise pas à extrapoler cette situation. Cependant rien ne permet d'exclure cette hypothèse.

\section{Usages de la parenté, transgression, actes de subversion}

Quoi qu'il en soit, la logique lignagère pouvait aussi être mobilisée pour subvertir les injonctions de la politique soviétique et servir les intérêts propres des lignages. La coïncidence entre une cellule du parti et un lignage devenait alors le support à tous les détournements, des priorités politiques comme des moyens matériels. Les cas de subversion étaient, non sans surprise, particulièrement courants. En tout cas, ils faisaient l'objet d'un grand nombre de rapports de la police politique et de plaintes permanentes pendant les réunions du parti en Kirghizie. En vertu des liens de parenté qui unissaient les dirigeants locaux à leurs administrés, les mesures d'imposition ou de confiscation, par exemple, n'étaient pas appliquées selon les règles attendues ${ }^{46}$. Les biens octroyés aux rajony ou encore aux kolkhozes étaient redistribués selon le principe lignager. Le président du kolkhoze Kzyl-Èmček, dans le district de Naryn, distribuait les rouleaux de tissus expédiés par le centre pour les kolkhoziens, aux seuls membres de son lignage ${ }^{47}$. Ces phénomènes illustraient à

46. CGA PD KR, 10/1/363/155, lettre au Comité du parti du rajon d'Alamedin, décembre 1931.

47. CGA KR, 23/1/845/78-93, «Doklad o bor’be s hišeniem i uničtoženiem skota » [Exposé sur la lutte contre le vol et la destruction du bétail],1934. 
nouveau la prééminence des solidarités de lignages sur celles de classes, confirmant que les divisions de classe ne recoupaient pratiquement jamais les divisions lignagères. Cette primauté du critère lignager dans la structuration de la société constituait de fait un obstacle puissant à une politique fondée sur la différenciation sociale. Dans ces régions, pendant la dékoulakisation (1929-1932), seule l'intervention de troïkas extérieures à ces logiques pouvait garantir l'application de la politique de répression des éleveurs les plus riches. En témoigne l'exemple d'un chef de lignage kirghiz qui avait échappé à plusieurs reprises à l'expropriation grâce à la complicité des membres et du président de son sel'sovet qui lui avaient fourni de fausses attestations. Il était enregistré comme bednjak, éleveur pauvre, alors qu'il ressortissait de la catégorie de bay-manap. Cet homme avait consolidé son « impunité » en promettant sa fille au responsable des collectes du sel'sovet avec lequel il entretenait ipso facto des liens intimes. On lit dans le rapport de son arrestation de 1931 dressée par l'OGPU qu'à l'issue de l'opération nocturne de dékoulakisation, les membres du sel'sovet dont le président, sont venus rendre hommage au riche éleveur kirghiz, l'ont embrassé et lui ont dit adieu sous les yeux de la troïka ${ }^{48}$.

La ressource que constitue le don d'une femme était d'un usage banal dans les sociétés d'Asie centrale, a fortiori en temps de crise. Au Kazakhstan, par exemple, dans l'aoul $\mathrm{n}^{\circ} 2$ du rajon de Malasar de l'okrug d'Alma-Ata, le plénipotentiaire responsable des collectes, voulant marier son fils, demanda à un homme pauvre de lui céder sa fille, en échange de quoi, en guise de kalym, il considérerait que cet homme avait rempli sa part du plan. Le kalym, c'est-à-dire les biens que devait fournir la famille du futur époux à celle de sa fiancée, était une condition sine qua non du mariage, que la loi soviétique avait interdit. La constitution du kalym demeurait cependant une préoccupation fondamentale pour les familles. L'acceptation, pour un foyer kazakh, de donner sa fille «gratuitement» en échange d'une simple exemption du plan montrait à quel point la pression des collectes était forte pour les familles, et révélait en même temps le pouvoir du plénipotentiaire, puissant jusqu'à obtenir des brus sans kalym. Ce cas de manipulation que l'OGPU mettait sur le compte de l'infiltration des bays dans les appareils n'était qu'une manifestation des nouveaux rapports de pouvoir en vigueur dans l'aoul ${ }^{49}$.

En plus des manquements à la doctrine, on recensait de nombreux actes subversifs sur le plan des mœurs de la part des dirigeants de rajon, de sel'́sovet et parfois d'oblast' qui étaient décrits et pensés comme des actes antisoviétiques, des « crimes de vie quotidienne » par les autorités républicaines du parti et l'OGPU. D'après ces dernières, ces actes engageaient la réputation du parti et du régime et justifiaient un renvoi et une exclusion du parti. On trouvait dans la liste des conduites condamna-

48. CGA PD KR, 10/1/363/87-89, «Doklad OGPU o predvaritel'nyh itogah po iz”jatiju kulacko-bajmanapskih hozjajstv » [Rapport de l'OGPU sur le bilan préliminaire des confiscations des familles de koulaks-baj-manap], juillet 1931.

49. Alexis Berelowitch, Viktor Danilov, Sovetskaja derevnja glazami VČK, OGPU, NKVD, dokumenty i materialy [La campagne soviétique vue par la VČK, l'OGPU et le NKVD, documents et matériaux], tome III (1930-1934), livre 1, 1930-1931, M. : ROSSPEN, 2003, p. 518 
bles, les attitudes rétrogrades envers les femmes et la propension à boire, qui n'étaient pas l'apanage des habitants d'Asie Centrale, mais aussi un certain nombre d'actes de sociabilité et de rituels sur lesquels reposaient justement l'autorité des élites politiques locales. Les membres des appareils organisaient et se rendaient à des festins honorifiques que leur clientèle leur destinait ${ }^{50}$; ils assistaient aux anniversaires de décès des membres de leur lignage qui comportaient non seulement de forts enjeux sociaux puisqu'ils faisaient intervenir les grandes lignes de descendance, les uruu, mais aussi une dimension religieuse; ils participaient aux jeux hippiques qui se tenaient l'été sur les hauts pâturages et qui étaient notamment l'occasion de régler diverses affaires (litiges, alliances, etc.). Les membres des appareils savaient qu'ils allaient à l'encontre des normes de conduite édictées par le parti mais ils étaient obligés de participer à ces rites qui intervenaient directement dans la cohésion des groupes lignagers. Leur présence aux rites était la condition de leur maintien et de leur intégration dans la société et leur assurait une légitimité qu'ils mettaient à profit dans les appareils ${ }^{51}$.

Le jeu des lignages dans les appareils soviétiques ne relevait pas nécessairement d'actes de résistance ou de contestation. Il était vraisemblablement davantage le résultat de pratiques séculaires et de principes d'organisation sociale et politique propres à ces sociétés. D'ailleurs, les formes de loyauté et de transgression n'étaient pas exclusives l'une de l'autre dans un même appareil. Au contraire, dans la plupart des cas, elles coexistaient et étaient fonction des circonstances et des personnes. Le fonctionnement des appareils locaux indigénisés au Kirghizstan peut ainsi être lu comme le fruit d'une instrumentalisation réciproque des pouvoirs soviétiques centraux (notamment républicains) et des lignages. Chacun utilise les moyens de légitimation que l'autre peut lui procurer. Les dirigeants locaux tirent de leur position dans l'appareil une puissance que leur confère le statut de relais de l'État dont la population a déjà éprouvé la capacité de violence et ce, depuis son intégration dans l'Empire tsariste. Les pouvoirs républicains reconnaissent implicitement à ceux qui se réclament d'une autorité lignagère une certaine efficacité à faire appliquer les directives et à être représentatifs pour les sociétés d'éleveurs. Cet équilibre est néanmoins précaire et susceptible d'être détruit par des mesures de purges et de neutralisation des lignages. Un dirigeant kirghiz du parti se plaignait, d'après le sténogramme d'un plénum du parti en 1932, que les luttes de clan à l'intérieur des appareils n'aient jamais été sanctionnées.

50. Les fêtes et les jeux traditionnels furent, dans l'après-guerre, non seulement tolérés par le régime mais instrumentalisés comme lieux d'intervention et de communication politique.

51. CGA PD KR, 10/1/155/127-134, procès-verbal de la session extraordinaire du collège du parti, village de Karabalta, comprenant les décisions prises à l'encontre de 8 membres du parti (kandidat) ou agents de l'État de petite catégorie (milicien) pour participation ou organisation de manifestations « traditionnelles », « aš » (funérailles) ou « toj » (festin notamment à l'occasion de circoncision ou de mariage). 


\section{3) La sédentarisation et la coercition collectiviste : une réponse à l'échec de l'indigénisation ?}

Même si le projet de collectivisation totale de l'agriculture relevait d'un programme pensé pour l'ensemble de l'URSS dans le cadre du Premier Plan quinquennal qui visait à mettre l'économie agricole au service du développement industriel accéléré, il doit être appréhendé tant à l'échelle des sociétés pastorales que paysannes des diverses régions de l'URSS, comme une atteinte délibérée au monde rural considéré par le pouvoir comme incontrôlable et responsable des crises d'approvisionnement de la fin des années 1920. La collectivisation et singulièrement les mesures de sédentarisation prennent place dans un continuum de répression qui s'accélère en 1928 avec le lancement de la campagne d'expropriation et de déportation des manap et des bays. Le sentiment de perte de contrôle de la société et des campagnes de la part du pouvoir, que l'autonomie des lignages au Kirghizstan notamment vient corroborer, apparaît comme l'une des hypothèses permettant d'expliquer d'une part la brutalité des conditions de mise en œuvre de la sédentarisation et, d'autre part, son impact sur la structure traditionnelle de la société et sur les prérogatives de ses institutions.

\section{La campagne de répression des élites traditionnelles}

Inhérente au projet révolutionnaire d'expropriation des classes dominantes au bénéfice des « paysans et des ouvriers », l'épuration des élites économiques et politiques était un invariant de la politique soviétique pré-stalinienne. Cependant, les modalités de sa réalisation se durcirent dès la crise des collectes qui marque la fin de la NEP et la consolidation du pouvoir de Stalin. Avant le lancement de la collectivisation totale, la liquidation des bays et des manap avait cours sous la forme d'une expropriation des biens d'une partie bien circonscrite de ce groupe social et de leur déplacement ${ }^{52}$. Le terme manap recouvrait en effet plusieurs réalités sociales et politiques dans la société kirghize. Son acception générique renvoie à une figure charismatique exerçant son autorité politique sur un groupe, laquelle autorité n'est pas fondée sur la richesse et la propriété mais sur la capacité à diriger, à résoudre les conflits et dans certains cas sur l'ascendance. L'aisance des manap était davantage le résultat de leur position qu'une condition de leur élection à la tête d'un groupe ${ }^{53}$. L'institution du manap existait donc à différents niveaux de la

52. CGA PD KR, 10/1/363/101a-101b, 20/08/1931, procès-verbal de réunion de la commission de la troïka du parti sur la confiscation des foyers koulaks, sur la base des matériaux rassemblés par l'OGPU, comprenant la liste pour le rajon de Džetyoguz des manap, leur appartenance lignagère, divers éléments biographiques, et chefs d'accusation. La grande majorité est condamnée comme «éléments socialement dangereux » à la confiscation des biens, au recouvrement de l'impôt dû et au déplacement.

53. Cf. les différentes acceptions relevées par le lexicographe K.K. Judahin, dans Kirgizskorusskij slovar' [Dictionnaire kirghiz-russe], M., 1985, t .2, p. 16. 
hiérarchie sociale puisque celui-ci pouvait être aussi bien à la tête d'un uruu, d'un el (groupe territorial à base généalogique), et dans la période coloniale, les čolok manap (littéralement petits manap) constituaient le creuset dans lequel étaient choisis les représentants des plus petites unités administratives, regroupant «50 familles » ${ }^{54}$. Ces figures, bien que confusément identifiées par le pouvoir soviétique, ont naturellement été considérées comme relevant de statuts contradictoires avec l'idéologie communiste mais plus encore comme des obstacles évidents à l'instauration du mode de gouvernement soviétique. Pour autant, seuls des manap qui pouvaient se prévaloir d'une ascendance prestigieuse et d'un charisme conséquent subirent, dans un premier temps, la déportation hors de Kirghizie, à Orenburg. C'était le cas des descendants du manap Šabdan Batyr et de ses fils qui avaient respectivement défendu le droit des Kirghiz à la terre occupée par les émigrés-paysans russes (1905-1912) et qui avaient soutenu le soulèvement populaire des Kirghiz contre la conscription décrétée par le tsar en juillet 191655. La confiscation des biens des bays et des manap demeurait la sanction la plus répandue adoptée à l'encontre de la majorité des représentants de l'ordre ancien plus nombreux dans la première décennie du régime soviétique qu'en 1928-1930.

Par une résolution du Comité central et du Conseil des commissaires du peuple de Kirghizie du 4 février 1929, prolongeant une politique à l'œuvre depuis plusieurs années, les bays et les manap définis comme «éléments socialement étrangers » devinrent la cible d'une répression spécifique destinée à la fois à purger toutes les instances politiques d'échelon inférieur et à préparer la sédentarisation. C'est là que se rejoignent la problématique du pouvoir local et celle de la sédentarisation. À la différence du programme de dékoulakisation entendu comme devant s'appliquer à la paysannerie européenne, la résolution stipulait en effet que seuls les districts nomades et semi-nomades étaient concernés par cette mesure et que devaient être confisqués et déportés hors de la république les éleveurs nomades possédant plus de 400 têtes de bétail et les semi-nomades en possession d'un cheptel de plus de 300 têtes. Par ailleurs, le statut social proprement dit pouvait suffire à justifier les mesures de répression destinées aux manap si la personne descendait en effet d'un manap, utilisait des prérogatives lignagères, avait occupé sous l'Empire des fonctions de chef de district (volost') ou de juge coutumier, avait été décoré par le gouvernement du tsar pour avoir mené une lutte désormais qualifiée d'antisoviétique ${ }^{56}$. La totalité des biens confisqués devait être redistribuée aux plus pauvres de l'aoul et en priorité aux personnes exploitées par le bay ou le manap, et collectivisée dans le cadre d'un kolkhoze, afin d'impliquer les « classes

54. Israilova-Har'ehuzen, Tradicionnoe obščestvo kyrgyzov v period russkoj kolonizacii..., p. 92 et suiv.

55. Cf. la série de documents d'archives publiés dans Šabdan Baatyr, èpoha i ličnost' [Šabdan Baatyr, une époque et une personnalité], Biškek : Šam, 1999.

56. CGA KR, 21/4/16, l'ensemble du dossier de l'année 1929 consigné dans le fonds du CIK, sur la déportation des bay-manap, comprenant les lettres de contestation des personnes concernées et déportées à Orenburg, les procès-verbaux des commissions régionales et de district d'identification et d'arrestation, les dénonciations collectives. 
dominées » dans cette procédure et, dans le même temps, de soutenir l'effort de collectivisation. Enfin, la confiscation et la déportation des bay-manap devaient avoir lieu en moins d'un mois et être supervisées par les organes de la police politique (OGPU) et de la milice, puis par les administrations de région et les représentants des organisations agricoles chargées de la collectivisation qui prendraient alors en main la répartition des biens ${ }^{57}$. Ces dernières devaient également organiser des commissions d'éleveurs pauvres appelés à dénoncer les bays exploiteurs et à participer à la répression ${ }^{58}$. Le texte exigeait de condamner en tout, pour l'ensemble de la Kirghizie, seulement 40 bay-manap et leur famille. Dans les faits, cette mesure ouvrait la porte à une répression plus générale et moins soucieuse des critères établis par les textes.

La violence de cette campagne d'élimination des manap apparaît en effet dans l'incompréhension par la population de ces mesures qui furent reçues comme une atteinte à la société pastorale et à l'identité lignagère, ainsi que le soulignent de nombreux documents d'archives ${ }^{59}$. Car le pouvoir formulait aussi ces intentions en termes de destruction des logiques claniques qui, de fait, régnaient dans les organes soviétiques d'échelon inférieur et intermédiaire et empêchaient la «construction kolkhozienne » et la « soviétisation de l'aoul». L'annonce du lancement de cette campagne, publiée quelques jours après le décret idoine dans le quotidien Sovetskaja Kirgizija, mais surtout le début des procédures de déportation, notamment vers l'Ukraine, provoquèrent une émigration significative des populations d'éleveurs nomades ${ }^{60}$. Par exemple, 300 familles environ quittèrent la vallée de l'Alaj pour la Chine ${ }^{61}$, des aouls de la région d'Atbaši se vidèrent partiellement, les uns et les autres emportant avec eux une relativement grande quantité de bétail (30 000 têtes d'ovins et 15000 têtes de gros bétail). La réaction des autorités du Parti communiste de Kirghizie ne se fit pas attendre : des inspecteurs spéciaux furent envoyés notamment dans les kanton de Naryn et de Talas où se réfugièrent certains bay-manap qui voulaient échapper à la répression ${ }^{62}$. Là encore, les sources montrent la solidarité de la population pour qui l'atteinte aux manap portait

57. CGA KR, 23/1/148/1-5, « Instrukcija CIK-a i SNK KirASSR “o porjadke likvidacii bajskih i kulackih hozjastv v rajonah splošnoj kollektivizacii” » [Instruction du Comité central et du Conseil des commissaires du peuple de la république autonome de Kirghizie sur "la procédure de liquidation des exploitations bays et koulaks dans les régions de collectivisation totale"], 25/ 12/1928 ; CGA PD RK, 10/1/363/87-89, Exposé de l'OGPU sur le bilan de la confiscation, 1931 ; CGA PD KR, 10/1/159/136 ; 138, Procès-verbal n 49 du Comité du parti sur la fuite des manap, 13/11/1928.

58. CGA PD KR, 10/1/352/75, Attestation sur la dénonciation d'un chef de lignage par un sel'sovet et un groupe de pauvres, 09/08/1931.

59. CGA PD KR, 10/1/370/8-17, Sténogramme du 4e Plenum du Comité kirghiz du parti communiste, $15 / 01 / 1932 /$

60. Le nombre de foyers nomades est passé de 8983 à 7501 entre 1931 et 1934 dans la région de Naryn, cf. CGA KR, 21/9/111/41-42.

61. CGA PD KR, 10/1/506/50-53, Note d'Isakeev au Sredazbjuro, 1933.

62. CGA PD KR, 10/1/155/138. 
«préjudice au lignage »63 et qui semblait peu sensible aux arguments de classe promus par le pouvoir soviétique, à l'exception de certains individus dont les intérêts et peut-être les convictions les poussaient à rallier le camp du pouvoir. Le mécontentement de la société était instrumentalisé à la fois par les victimes désignées de la campagne de répression, les «bay-manap » qui, là encore, invoquaient la parenté commune pour rallier les aouls de leur région ${ }^{64}$, comme par la hiérarchie qui interprétait les faits de protestation comme le résultat de rivalités lignagères plutôt que comme la conséquence de la répression et de la campagne de collectivisation qui avait lieu en même temps. Pourtant, les appareils intermédiaires euxmêmes, - et ce fut le cas du comité exécutif de kanton de Naryn — formulaient des recours auprès du Comité central exécutif de la République et demandaient l'annulation de la confiscation et de la déportation de certaines figures ${ }^{65}$. À ces plaintes, le pouvoir central répondait, selon les circonstances, par un refus ou une demande d'explication pouvant aboutir à la suppression de la peine.

\section{La sédentarisation : de l'idéologie au chaos de la mise en œuvre}

C'est dans ce climat qu'intervinrent les mesures de sédentarisation et de collectivisation. Ainsi trois programmes distincts allaient être conduits de concert, la répression des élites rurales, la collectivisation et la sédentarisation appartenant en effet à la même séquence chronologique et au même projet idéologique : la « soviétisation » de la société pastorale kirghize. Cette corrélation des trois processus ne relevait pas seulement d'une concordance de calendrier mais aussi de l'idéologie qui animait les dirigeants bolcheviks qui se trouvaient à la tête des républiques ou des régions peuplées de nomades en URSS. Dans la vulgate évolutionniste marxiste des stades de développement des sociétés qui constituait leur référence, le nomadisme était associé à un stade de développement inférieur reposant sur une structure sociale «féodalo-patriarcale » incarnée par l'organisation lignagère. Dans ce modèle, la structure sociale, soit le système d'institutions et de valeurs, fonctionnait en interdépendance avec le mode de production, c'est-à-dire la base économique. Autrement dit, en termes marxistes, superstructure et infrastructure procédaient réciproquement l'une de l'autre. Le corollaire immédiat de cette théorie faisait qu'en agissant sur l'un des termes on pouvait transformer l'autre. Ainsi, on pouvait aboutir à la sédentarisation en intervenant à la fois sur l'élevage pastoral et sur la structure lignagère. Les deux aspects de la sédentarisation en faisant dès lors un programme de transformation sociale totale.

63. CGA PD KR, 10/1/201/29-30, Procès-verbal nº 8 sur la déportation des bay-manap et la résistance de la population, 11/03/1929.

64. CGA PD KR, 10/1/307/144-146, Procès-verbal n 66, « Informacija komissii Sredazbjuro Ck VKP(b) o rezul'tatah izučenija položenija v Narynskom i atbašinskom rajonah » [Information de la commission du bureau d'Asie centrale du Comité central du parti sur les résultats de l'étude de la situation des districts de Naryn et d'Atbaši], 13/07/1931.

65. CGA PD KR, 10/1/517/53-64, Note du responsable de la GPU de Kirghizie, 1933. 
Dans cette perspective, la sédentarisation devait permettre la modernisation du mode de production des sociétés nomades et la disparition des rapports lignagers au profit de rapports de classe fondés sur la division sociale du travail. La modernisation de l'économie de ces sociétés voulues par le projet soviétique ne préjugeait pas de la disparition de l'élevage comme activité principale, pas même de celle du pastoralisme. La sédentarisation signifiait certes, mais pas exclusivement ni obligatoirement, la fixation des populations mais surtout elle avait pour objectif de briser le rapport de dépendance entre société et cheptel, de faire en sorte que l'ensemble d'un groupe humain ne soit plus assujetti au déplacement pastoral, que l'élevage n'occupe plus l'ensemble du groupe. À terme, la sédentarisation devait conduire à ce que le pastoralisme n'organise plus la vision du monde des sociétés anciennement nomades. Ainsi, cette conception admettait implicitement - et de plus en plus explicitement à mesure que l'expérience acculait les autorités soviétiques à plus de modestie - la possibilité d'une mobilité pastorale à condition qu'elle soit spécialisée et professionnalisée et qu'elle n'entre pas en contradiction avec la finalité de la modernisation. La fixation des hommes, dans cette idéologie, devait concerner tous ceux qui n'étaient pas directement indispensables au pacage des troupeaux, soit la grande majorité du groupe.

Par ailleurs, la sédentarisation impliquait, d'une part, la collectivisation des moyens et des biens de production à laquelle elle tenait lieu de préalable, d'autre part, la réorganisation du travail agricole (par la répartition des tâches, la professionnalisation, la technicisation, le productivisme, etc.) et de son encadrement social. En somme, aux yeux du projet soviétique, la sédentarisation était un processus de passage d'un état à un autre, d'un stade de développement à un autre qui supposait une action politique sur le corps social. Elle représentait une forme spécifique de la modernisation planifiée. Son ambition totalisante expliquait ainsi la capacité de cette politique à affecter profondément les institutions sociales traditionnelles, d'autant plus qu'elle se déroula dans le contexte répressif de la collectivisation.

Alors que le mot d'ordre de sédentarisation fut lancé en février 1929, le plan ad hoc pour la Kirghizie fut conçu en 1931 et organisé pour suivre le calendrier suivant pour les 12 rajony d'élevage nomade dits «de sédentarisation » : 10000 foyers devaient être sédentarisés en 1931, 30000 foyers en 1932, 40000 en 1933, les 20000 restants appartenant à d'autres rajony non exclusivement nomades ${ }^{66}$. Ces normes furent décidées par un appareil créé spécialement pour conduire la sédentarisation à la suite d'une résolution du 3 avril $1931^{67}$ : le Comité de sédentarisation. Cet organe se composait du président du Conseil des commissaires du peuple de la RSSA kirghize, Abdrahmanov, du vice-président, Ščerbakov, de membres des commissariats à l'Agriculture et aux Finances, du vice-président du Gosplan de Kirghizie, de responsables de la Direction de l'eau et du président du kolhozcentr

66. CGA RK, 328/1/985/2, «Plan rabot komissii po osedaniju pri Kirkolhozcentre » [Plan de travail de la commission de sédentarisation du centre pour les kolkhozes de Kirghizie], du 25/ 05/1932 au 02/12/1932.

67. Džumadil S. Baktygulov, Socialističeskoe preobrazovanie kirgizskogo aula (1928-1940) [La transformation socialiste de l'aoul kirghiz], Frunze : Izd-vo Ilim, 1978, p. 123. 
(administration chargée de la collectivisation et des kolkhozes). Bien que les plus hautes autorités de l'État kirghiz aient été choisies pour présider cet organe et lui conférer une force et une légitimité, son activité réelle et l'implication de ses membres demeurèrent quasiment nulles au moins jusqu'à la fin de l'année 1932. Le président du Conseil des commissaires du peuple de la RSSA, comme les autres membres, était davantage mobilisé par l'urgence du plan de collectivisation des piémonts céréaliers et des régions occupées par les populations d'origine européenne, et le comité ne put se réunir que trois fois en 1931, deux fois en 1932 et une fois en 1933.

La faiblesse de la direction du comité de sédentarisation explique en partie les problèmes rencontrés sur le terrain par les cadres subalternes chargés d'appliquer des instructions pour lesquelles ils avaient peu de moyens et qui n'étaient ni contrôlées ni reconnues par l'appareil central. Le plus souvent, des cadres peu ou pas qualifiés avaient des charges trop importantes au regard de leurs compétences comme de choisir les lieux d'implantation des exploitations, de superviser leur construction, de procéder à la distribution des terres, etc. Localement, les organisations agricoles en charge de la sédentarisation manquaient d'agronomes et de zootechniciens, en étaient conscients et demandaient sans cesse au centre qu'il remédie à cette situation, mais sans succès ${ }^{68}$. L'assignation des populations dans des lieux inappropriés conduisit régulièrement à la désertion de ces nouveaux villages voire à leur destruction par les groupes d'éleveurs, comme ce fut le cas dans le district d'Atbaši ${ }^{69}$. Ce phénomène, noté également au Kazakhstan dès 1929 , peut être identifié comme une première étape de sédentarisation (année 1931) marquée par l'échec des initiatives coercitives locales, par une mauvaise sélection des lieux de sédentarisation souvent impropres à conduire toute activité productive.

Dans un deuxième temps, le programme de sédentarisation de plus en plus exigeant fut confronté à différents problèmes matériels, liés à la fois au manque de moyens généralisé à cette époque en URSS et à la forte bureaucratisation des structures chargées de la sédentarisation. Le budget théoriquement imparti à la sédentarisation ne fut pas entièrement délivré par la RSSA au comité. Les commissariats à l'Agriculture et aux Finances n'organisèrent pas rigoureusement le financement de la sédentarisation; les organisations sur le terrain souffraient d'une pénurie patente de matériaux de construction destinés aux villages de sédentarisation (maisons et bâtiments agricoles). D'après les analyses des appareils de direction du centre, l'absence d'encadrement social (pas de ravitaillement alimentaire, pas de service de santé, etc.) dissuadait les populations en voie de sédentarisation de se soumettre

68. CGA PD KR, 10/1/517/113-125, « Specsvodka GPU » [Rapport spécial de la GPU], 21/03/ 1933. CGA KR, 382/1/213/43, « Protokol n 35 očerednogo zasedanija Pravlenija Narynskogo Kantkolhozsojuza “o provedenii čistki i otčetnyh predvybornoj kampanii v kolhozah" »,06/01/ 1930.

69. CGA KR, 23/1/780/31-35, «Vypiski iz protokola ${ }^{\circ} 183$ zasedanija bjuro Kirobkoma VKP(b) o hode osedanija v Atbašinskom i Alaj-Gul'činskom rajone » [Note d'après le procèsverbal n 183 de la réunion du bureau du Comité kirghiz du parti sur le déroulement de la sédentarisation dans les districts d'Atbaši et d'Alaj-Gulč́i], 16/12/1933. 
au projet. Enfin, la faiblesse voire l'inexistence de recherches scientifiques sur l'organisation potentielle de l'économie dans les régions d'élevage expliquait aux yeux des dirigeants l'échec de la sédentarisation ${ }^{70}$.

\section{De l'imbrication des politiques d'épuration des chefs locaux et de la sédentarisation-collectivisation}

Ce sont pourtant les conséquences de ces politiques, - la famine, le mouvement d'émigration vers la Chine et la chute du cheptel de près de $50 \% \%^{71}$ - qui, associées au régime de coercition de la collectivisation, compliquèrent la mise en œuvre de ce projet tout en affectant les institutions lignagères. En effet, les stratégies de contestation déployées par les appareils locaux contre les réquisitions de denrées confortèrent les agents chargés de la collectivisation et de la sédentarisation dans leur usage de la répression. Ces représentants de la police politique, extérieurs aux appareils dans lesquels ils devaient exercer, se heurtaient à de véritables bastions clientélistes. Par exemple, en 1932, le secrétaire du rajkom de Naryn, un Kirghiz originaire de Karakol se comportait dans le rajon en chef absolu, défiait ouvertement les directives du centre et décidait seul des mesures à appliquer. Notamment, il interdisait à tous ses subordonnés d'engager les collectes de céréales sans son aval, et refusait que l'OGPU, traditionnellement en charge du contrôle des livraisons obligatoires, intervienne malgré le visa des autorités centrales ${ }^{72}$. Cette politique recevait le soutien de la population que les ponctions régulières de denrées portaient au bord de la famine. Le secrétaire de rajkom était toutefois accusé d'abus de pouvoir en tout genre : corruption, licenciements gratuits, favoritisme vis-à-vis des éleveurs les plus riches, nationalisme, etc. Son attitude face à l'OGPU, seul organe local susceptible de contrebalancer son pouvoir contribuait à discréditer le pouvoir central aux yeux de la population du rajon. De même, ils fustigeaient la présence de Kazakhs parmi les cadres techniques (agronomes, instructeurs) du rajon, attisant ainsi l'animosité des habitants envers les représentants étrangers présents dans les instances de pouvoir. Selon la même logique d'appartenance lignagère commune, le procureur du rajon de Naryn accordait des traitements de faveur variés aux membres condamnés de son clan, notamment en les acquittant et leur évitant le versement $\mathrm{d}^{\prime}$ amendes $^{73}$. Ce type de faits, mis en évidence régulièrement par la police politique, entraîna en temps de collectivisation, une recrudescence des campagnes de purges à tous les niveaux. En cela, cette période de sédentarisation est cruciale pour comprendre l'étiolement des lignages comme socle de la vie politique.

70. CGA PD KR, 10/1/370/155.

71. CGA KR, 21/9/111/40-57, « Otčetnyj doklad o rabote Narynskogo rajispolkoma KirASSR za 1931-1934 », qui montre notamment que le cheptel, dans le rajon de Naryn est passé de 303356 à 149008 têtes de bétail entre 1931 et 1934 .

72. CGA PD KR, 10/1/433/54-66, Rapport de l'OGPU au Comité kirghiz du parti, 19/11/1932.

73. CGA PD KR, 10/1/308/112-113, Procès-verbal n81, 17/11/1931. 
Ainsi, à l'échelle d'un sel'sovet et d'un kolkhoze, en l'occurrence dans le sel'sovet de Minbulak du district de Naryn, une brigade fut chargée de purger l'administration locale ainsi que celle des kolkhozes. Pendant la procédure, la brigade découvrit en sus de ses soupçons préalables que l'administration avait classé comme bay-manap des éleveurs pauvres ou moyens, dans le but de les neutraliser, tandis qu'inversement certains bay-manap théoriquement soumis à la confiscation et à la privation de leurs droits civiques étaient catégorisés comme pauvres ou moyens, pouvant ainsi se présenter aux élections locales et exercer leur droit de vote. D'autre part, le président du sel'sovet était le fils du mollah local, cette situation étant connue et couverte par le secrétaire du rajkom de Naryn. À l'échelle de la direction des kolkhozes du même district, plusieurs faits dénotaient le caractère généralisé de l'insubordination envers le pouvoir, la connaissance que les autorités centrales avaient d'une situation qu'elles appréhendaient, comme partout en URSS, comme le signe d'un désordre incontrôlable voire du chaos ${ }^{74}$. Le secrétaire du kolkhoze Džany-žalyp du sel'sovet d'Aktala menait par exemple, une campagne de désinformation incitant les kolkhoziens à se préparer à l'insurrection contre le pouvoir soviétique, en exploitant la menace d'entrée en guerre de l'URSS contre le Japon et en faisant croire à la mort des hauts dirigeants kirghiz à Frunze.

À la seule échelle du district de Naryn, depuis les cadres de direction du district jusqu'aux kolkhozes, les éléments de transgression et de résistance recueillis par la police politique, bien que leur abondance et leur concentration soient susceptibles de produire une image extrapolée de la réalité, n'en révèlent pas moins à quel point le pouvoir était inquiet des conditions de mise en œuvre de la triple campagne de répression des bay-manap, de collectivisation et de sédentarisation et se donnait les raisons d'une coercition particulièrement violente.

\section{Conclusion}

Le climat de répression général et en particulier les purges régulières des appareils locaux, les collectes obligatoires opérées par des acteurs extérieurs à la société locale, à savoir par les agents de la police politique envoyés pour l'occasion, sont sans nul doute à l'origine d'une vague de fuite massive de la population parmi laquelle on comptait notamment les éleveurs dits riches, les personnes que leur autorité sociale rendait suspectes aux yeux du régime et enfin tous ceux qui, soumis dans un premier temps aux premières mesures de sédentarisation, tentèrent d'échapper au grand dénuement et à la famine qui caractérisaient la vie quotidienne des premiers villages en dur et des premiers kolkhozes ${ }^{75}$.

74. Nicolas Werth, « Les formes d'autonomie de la "société socialiste” », in Henry Rousso, éd., Stalinisme et nazisme : Histoire et mémoire comparée, Bruxelles : Éditions Complexe IHTPCNRS, 1999, p. 145-184.

75. CGA PD KR, 10/1/517/113-125, «Specsvodka o hode kampanii podgotovki k vesennej posevnoj » [Rapport spécial sur le déroulement de la campagne de préparation aux semailles de printemps], 21/03/1933. 
L'impact sur les lignages de ces départs vers la Chine et de la mortalité causée par la famine ou par la dégradation générale des conditions de vie se traduisit par le décès prématuré des plus âgés, c'est-à-dire des membres de la société détenteurs des savoirs pastoraux et lignagers. La dispersion géographique des groupes, non seulement vers la Chine mais également sur le territoire de la Kirghizie, mit elle aussi à l'épreuve la cohésion des unités sociales, que l'on pense ici aux aouls ou aux lignages. Mais plus encore, la reconfiguration du territoire pastoral opérée par la politique de sédentarisation et de collectivisation, qui consistait à la fois à exploiter les pratiques ancestrales de pacage sur les pâturages saisonniers et à développer les cultures fourragères, transforma l'assise socio-économique du pastoralisme. En impliquant une professionnalisation de la mobilité et donc de la fonction de berger et en procédant à la compartimentation du procès pastoral en différentes tâches spécialisées et technicisées, elle octroyait indéniablement une place de premier ordre à la structure du kolkhoze comme socle économique du pastoralisme au détriment des unités de parenté. Ces dernières ne perdaient pas la totalité de leurs prérogatives puisqu'elles pouvaient légalement disposer d'un cheptel mais elles n'étaient plus en capacité d'en assurer l'exploitation privée ni de contrôler par elles-mêmes la totalité des actions menées sur lui. Cette situation ôtait aux structures sociales traditionnelles (aouls, lignages, groupes de descendance) une part de leur matérialité économique et affaiblissait en cela leur poids dans la société. Si ce constat vaut comme schéma dans une configuration où au sein du kolkhoze sont absents tous types de liens de parenté entre l'ensemble des membres et notamment entre les membres dirigeants et les kolkhoziens ordinaires, il est soumis à de fortes nuances dans la mesure où à l'intérieur de la ferme collective se retrouvaient ou se recomposaient des unités sociales traditionnelles susceptibles d'exploiter les outils politiques et économiques du kolkhoze au profit de leur cohésion et de leur autorité. Mais cet aspect important de l'histoire reste peu exploré et difficile ici à démontrer rigoureusement.

Pour autant, peut-on en conclure que les lignages ont été véritablement écartés de la sphère politique ? Le système soviétique a-t-il opéré un changement majeur par rapport à l'expérience coloniale quant à la place tenue par les groupes de parenté dans la structure administrative et politique de la Kirghizie ? On ne peut écarter l'hypothèse d'une certaine continuité avec la situation antérieure telle qu'elle s'est constituée à l'époque tsariste. Durant la conquête de l'Asie centrale, les colonisateurs russes avaient déjà largement exploité l'organisation des sociétés segmentaires, en particulier des Kazakhs et des Kirghiz. Faute de pouvoir saisir un centre politique en effet inexistant, ils ont soumis successivement élément par élément de la structure segmentaire ${ }^{76}$, sans nécessairement tenir compte des hiérarchies locales. L'administration impériale, avec la promulgation en 1822 de la Charte des Kirghiz de Sibérie, légiféra par exemple sur l'institution kazakhe de la horde $(\check{z} \sharp z)$ afin de l'utiliser comme cadre de construction du pouvoir colonial,

76. Cf. Nurbulat Masanov et alii, Istorija Kazahstana: Narody i kul'tury [Histoire du Kazakhstan : peuples et cultures], Almaty : Daïk press, 2001,p. 158-177. 
alors même qu'elle en avait aboli la dignité de khan, la plus haute instance politique. Elle continuait à reconnaître la réalité de la Moyenne žtz en promouvant ses chefs locaux aux différents niveaux de la nouvelle hiérarchie administrative. Elle prévoyait en effet la création de charges d'okrug, de volost' et d'aoul sous la responsabilité d'un «sultan », référant directement à l'institution kazakhe qui désignait les personnes de descendance gengiskhanide seules habilitées à occuper la fonction de khan, de sultan ou de bij (juge) en raison de leur filiation. La formation du territoire des okruga se fondait, elle, sur l'identification des lieux d'hivernage (kstau) des Kazakhs. De sorte que la définition des frontières des okruga résultait d'une vision organisée du territoire des nomades. Les populations vouées à être recensées et administrées par l'okrug étaient identifiées comme ayant coutume d'hiverner dans les limites territoriales de la nouvelle division administrative, même si elles pouvaient en sortir pour rejoindre leurs pâturages de printemps et d'été. Ipso facto, l'okrug tenait également compte des divisions lignagères de la société liées à la répartition des stations saisonnières, et faisait coïncider dans une certaine mesure l'organisation sociale et la parenté avec les territoires administratifs.

Conforme à sa logique de création de statuts différenciés ${ }^{77}$, la politique coloniale qui a consisté à intégrer les élites lignagères dans son dispositif de direction en les gratifiant de privilèges particuliers $\mathrm{a}$, in fine, eu pour effet l'institutionnalisation administrative et bureaucratique des autorités traditionnelles. Le pouvoir colonial, conscient des conséquences de cette politique de conquête confortée par le Gouverneur de Sibérie occidentale, M.M. Speranskij dès 1822, dans les steppes, avait même cherché à profiter de la grande réforme de 1867 pour remodeler le maillage territorial et minorer le pouvoir des groupes de parenté sur les petites unités administratives. Pourtant, les documents décrivant les représentations locales de l'Empire dans les steppes kazakhes et les montagnes kirghizes, constitués principalement des rapports commandés par la Société impériale russe de géographie et des études de la Direction des migrations parues dans le cadre de la réforme de Stolypin, sont unanimes en ce qu'ils soulignent la présence et la puissance des lignages à l'échelle des volosti en particulier. Les volosti étaient non seulement dominés et dirigés par un lignage dont le représentant était accrédité par l'administration coloniale ${ }^{78}$, mais ils en portaient jusqu'au nom, cette situation se rencontrant

77. Cf. Adeeb Khalid « Backwardness and the Quest for Civilization: Early Soviet Central Asia in Comparative Perspective », Slavic Review, 2, 2006, p. 231-251.

78. Olga Brusina, «Obyčnoe pravo kočevogo naselenija Turkestana v sisteme rossijskogo upravlenija » [Le droit coutumier chez la population nomade du Turkestan dans le système de gouvernement de l'Empire russe], Sredneaziatskij etnografičeskij sbornik, 2006, p. 227. 
aussi bien en 1844 dans les steppes kazakhes ${ }^{79}$ que plus au sud chez les Kirghiz en $1889^{80}$, comme en 1912-1913 dans le Semireč $\mathrm{e}^{81}$.

Grodekov, juriste russe, missionné pour faire état des pratiques juridiques dans les régions kazakhes et kirghizes de l'oblast' du Syr-Daria, rapportait que la primauté d'un groupe de filiation se maintenait dans les régions les plus homogènes sur le plan lignager et profitait des caractéristiques du système électoral en vigueur. Les figures les plus charismatiques parvenaient à se faire élire sur une base lignagère et à être reconnues par l'administration coloniale. D'une part, leur appartenance à une lignée dominante et l'occupation antérieure de fonctions politiques les prédisposaient naturellement au rôle d'élu, pouvant légitimement prétendre à une fonction de représentation politique. D'autre part, le caractère mal défini des frontières de volost' et d'aoul et par conséquent leur manque de pertinence jouaient en faveur du pouvoir des chefs de lignages, qui pouvaient recueillir des voix de toutes parts en dépit des limites administratives. D'après les propos recueillis par Grodekov auprès des administrateurs russes des okruga de Čimkent et de Perovsk ${ }^{82}$, une nouvelle petite aristocratie lignagère composée des figures dirigeantes des volosti, des aouls et même des unités de 50 familles jouissait non seulement de son autorité traditionnelle mais des prérogatives induites par sa fonction dans le système colonial. Leur loyauté envers le tsar et l'Empire est, elle, plus difficile à analyser. Si on la mesure à l'aune des réactions de ces représentants locaux à l'ordre de conscription obligatoire de 1916, force est de constater la diversité des positions. D'après les documents d'archives publiés ${ }^{83}$, on comptait aussi bien des chefs de volost' soumis à l'autorité suprême qui s'efforcèrent, avec ou sans l'aide des Cosaques, de faire respecter l'ukaz et de constituer des listes d'hommes susceptibles de partir pour l'arrière, que des dirigeants indigènes soutenant ou prenant la tête de la rébellion. On peut même affirmer que les représentants autochtones de l'Empire ont pour la plupart tenté de convaincre leurs administrés de se plier à l'ordre de conscription. Quoi qu'il en soit,

79. «Svedenija o volostjah Kokpektinskogo okruga po isčisleniju, proizvedennomu v 1844 zasedatelem Suhomlinovym » [Données sur le volosti de l'okrug de kokpek d'après le recensement effectué par l'assesseur Suhomlinov en 1844], texte complet publié p. 70-80 par A. Konšin, «Materialy dlja istorii Stepnogo kraja IV. K istorii otkrytija Kokpektinskogo okruga, s priloženijami » in Zapiski Semipalatinskogo podotdela zapadno-sibirskogo otdela Imperatorskogo Russkogo Geografičeskogo obščestva [«Matériaux pour l'histoire des steppes IV. Histoire de la découverte de l'okrug de Kokpek et annexes » in Journal de la section de Semipalatinsk, département de Sibérie occidentale, de la Société impériale russe de géographie], volume II, Semipalatinsk, 1905, p. 1-127.

80. Nikolaj Grodekov, Kirgizy i karakirgizy Syr-darinskoj oblasti [Les Kirghiz et les Karakirghiz de l'oblast' du Syr-Daria], t. 1, Juridičeskij byt [Les usages juridiques], Tachkent, 1889, p. 13-15.

81. Materialy po obsledovaniju tuzemnogo i russkogo starožil'českogo hozjajstva i zemlepol'zovanija v Semireč enskoj oblasti [Matériaux de recherche sur les foyers indigènes et russes natifs et leurs usages de la terre dans l'oblast' du Semireč é, uezg de Vernyj], sost. Petr Rumjancev; Pereselen. Upravl. Gl. Upravl. Zemleustrojstva i Zemledelija. - t.4: Vernenskij uezd, $\mathrm{SPb}, 1913$.

82. Grodekov, Kirgizy i karakirgizy...,p. 17, 20.

83. Qaharly 1916 žyl (Qužattar men materialdar žingag்y) [La terrible année 1916 (Recueil de documents et de matériaux)], tome 1, Almaty, 1998, Qazaqstan, 424 p. et tome 2, 248 p. 
même si les écrits sur la vie politique des confins kazakhs et kirghiz, tenaient par principe une position critique envers la coïncidence des volosti avec les lignages et son potentiel d'insubordination, l'administration n'en prenait pas moins acte. Le caractère connu et très documenté du rôle des lignages dans la vie politique et économiques des sociétés nomades d'Asie centrale dans le cadre même du système colonial ${ }^{84}$ explique que les bolcheviks se soient très vite emparés de cette question et en aient fait un véritable enjeu de surveillance et de répression.

Les formes d'autonomie politique autochtones et les formes d'inertie qu'impliquait la présence des lignages dans les appareils locaux ont en effet justifié, aux yeux du pouvoir soviétique à l'échelle républicaine et centrale, la violence des transformations du Premier Plan quinquennal. Les purges des années de collectivisation et de sédentarisation peuvent ainsi être lues comme un retour sur la politique d'indigénisation dont le pouvoir avait, à la fin des années 1920 et au début des années 1930, éprouvé les risques qu'elle comportait, notamment celui de ne pas maîtriser le recrutement des nouveaux cadres autochtones. La campagne de répression des bays et des manap dirigée contre les «éléments socialement étrangers » ou «nuisibles » susceptibles de pénétrer l'appareil soviétique, si elle n'était pas conduite explicitement en relation avec l'indigénisation, s'inscrivait dans le même dispositif global visant à créer une société nouvelle, débarrassée des « exploiteurs » et promouvant l'initiative et la responsabilité des «dominés ». Pourtant une structuration des appareils en fonction des relations de parenté dans les régions d'élevage à forte majorité kirghize, telle que nous l'avons montrée, même si elle était précaire du fait de l'hostilité du régime à ce fonctionnement, pose la question de l'adaptation réciproque des logiques soviétiques et des logiques propres aux cultures politiques autochtones, puis celle de la combinaison des formes de légitimité et de loyauté. En dépit des purges et des différents phénomènes qui ont contribué à affaiblir les éléments de fonctionnement politique autochtones, il semble que l'hybridité politique qui est née de la rencontre entre deux formes de pouvoir et de loyauté a structuré durablement les pouvoirs locaux en Kirghizie, mettant parfois en échec le projet et l'idéal étatique soviétique ou au contraire garantissant sa mise en œuvre. On peut formuler l'hypothèse que ces pratiques ont contribué à enraciner le système soviétique dans les sociétés segmentaires et ont constitué des formes d'appropriation du nouveau régime, au moins dans une première étape de construction des pouvoirs circonscrite à l'entre-deux-guerres. Au cours de l'histoire soviétique de la Kirghizie, le contenu social des lignages a bien entendu évolué autant que son investissement politique par la société. Enfin, si l'on considère cette question sur un plus long terme et à un autre niveau de pouvoir, les

84. Voir notamment l'extraordinaire richesse des matériaux issus des expéditions Ščerbin et Rumjancev commanditées par la commission des migrations, respectivement en 1896-1900 et 1910-1913. Materialy po obsledovaniju..., op. cit., 1910-1913. Materialy po kirgizskomu zemlepol'zovaniju, sobrannye i razrabotannye ekspediciej po issledovaniju stepnyh oblastej [Matériaux sur l'usage des terres des Kirghiz, recueillis et traités par l'expédition de recherches sur les oblasti des steppes], G.U.Z.iZ. Pereselen. Upravl. 1898-1908. 
caractéristiques de gouvernement que l'on prête à la période durant laquelle T. Usubaliev dirigea le Parti communiste de Kirghizie entre 1961 et 1985, interroge plus structurellement encore les mécanismes d'interaction entre les normes du système politique soviétique et les logiques qui se réclament de la parenté.

CNRS - École des hautes études en sciences sociales

UMR 8083, centre d'études des mondes russe, caucasien et centre-européen

isabelle.ohayon@cercec.cnrs.fr 


\section{Commentaires du schéma de parenté «Schéma génétique de la tribu Sarybagiš, division Manap, branche èsengul $\gg 85$}

La figure reproduite ici à l'identique représente le schéma de parenté d'un lignage kirghiz, l'uruu èsengul, tel qu'il a été constitué par un informateur d'origine européenne à destination de l'OGPU en 1927. En effet, trouvé dans un dossier de correspondance entre la police politique et le parti, dans les Archives de documentation politique de la République kirghize (anciennes archives du parti communiste), ce schéma prend place dans une série de rapports dénonçant le pouvoir des lignages dans les instances locales. Cependant, nous ne disposons pas d'éléments de contextualisation plus précis qui permettraient de décrire l'usage concret qui en a été fait.

Dans sa forme originale, ce schéma occupe huit feuilles A4 et fait apparaître à côté de chaque symbole utilisé (cf. légende ou sa traduction infra ${ }^{86}$ ) le nom porté par le segment de parenté en question. Pour des raisons de lisibilité, ces noms, sauf pour les plus importants, n'ont pas été restitués ici. Ce schéma peut se lire en deux parties, telles qu'elles ont été distinguées dans les deux pages suivantes (feuille A et B). La première partie replace le segment èsengul dans la généalogie générale des lignages et des tribus kirghizes, en l'occurrence dans sa filiation avec la grande tribu Sarybagiš qui fait partie du groupe des Tagaj appartenant lui-même à « l'aile droite » (On), l'un des deux grands segments qui composent l'ethnie kirghize. Cette partie rend également compte de la relation de filiation qui lie le lignage èsengul à d'autres lignages de même rang et par conséquent à d'autres volost'.

La seconde partie décrit le segment dans ses ramifications contemporaines telles qu'elles se déploient à l'échelle du volost'éponyme èsengul en 1927. Les branches les plus développées montrent les relations qui unissent les quelque cent foyers qui habitent le district. Ainsi, les chiffres notés à côté de chaque point désignent l'âge des hommes vivants, lesquels se trouvent pour la plupart à la tête d'une famille. L'ensemble du schéma transcrit exclusivement la filiation patrilinéaire (tous les points renvoient à des figures masculines) et ne fait intervenir la mention des épouses qu'en cas de ménage polygame. Ainsi, la croix (+) ne désigne pas particulièrement l'individu de sexe féminin mais le mariage en contexte polygame.

85. CGA PD KR, 10/1/144/193.

86. Traduction contextuelle de la légende :

Plemja : ethnie ; plemennoe razdelenie : division ethnique ; rod : tribu; koleno : ramification ;

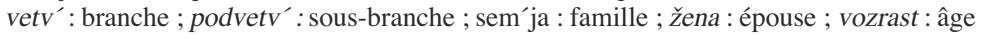




\section{GENETIČESKAJA SHEMA RODA SARYBAGIŠ, KOLENO MANAP} VETV' ĖSENGUL

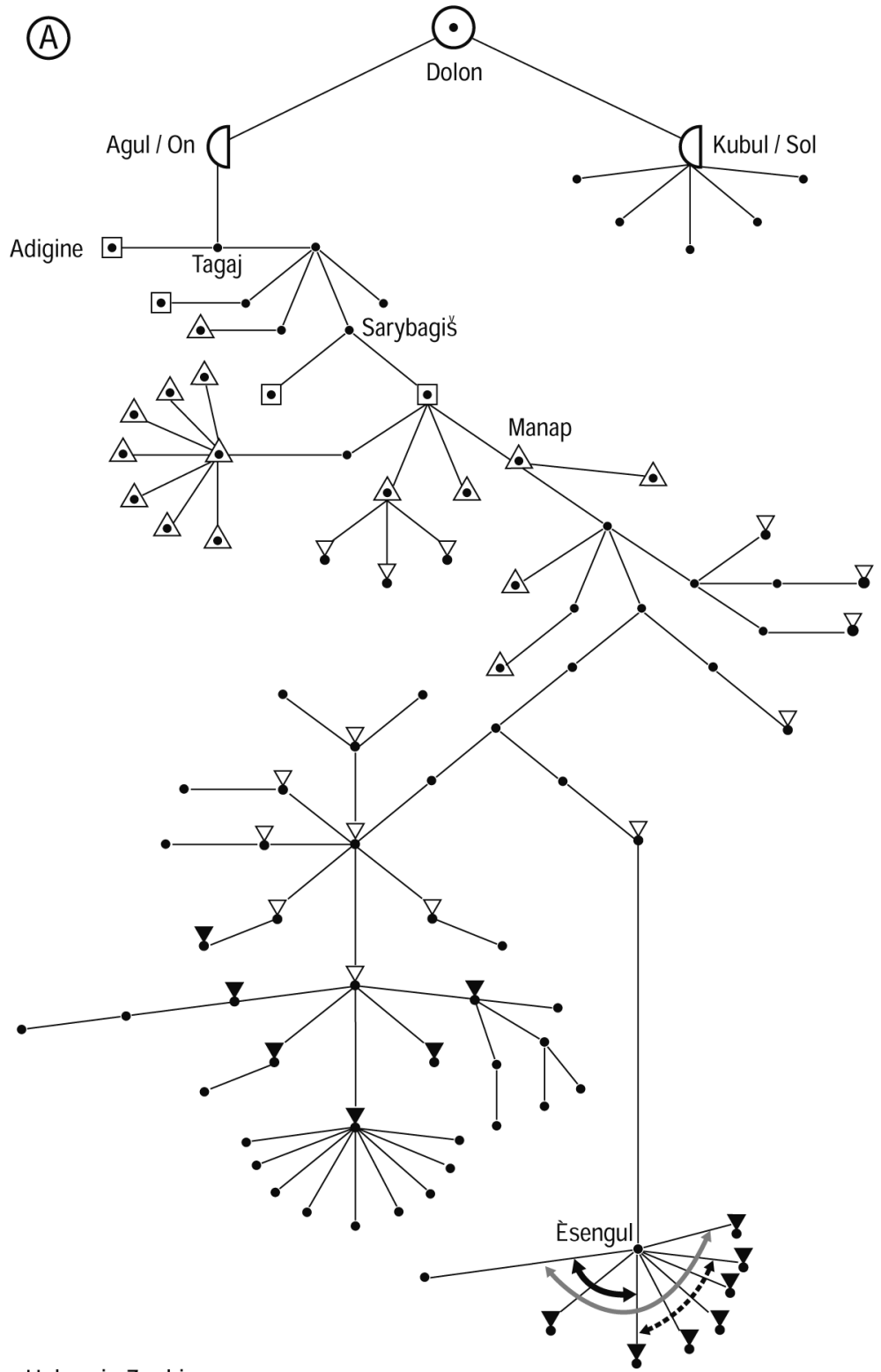

Uslovnie Znaki
(-) Plemja
Plemennoe razdelenie (On-Sol)
- Rod
๑) Koleno
8 Vetv'
$\gamma$ Podvetv'
- Sem'ja
-.ं- Žena
Sakizbek
- Décédé
20 Vozrast

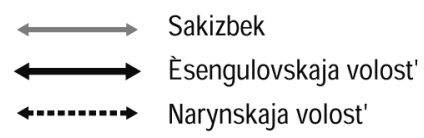

Julien Thorez, CNRS, Mondes iranien et indien, 2008 


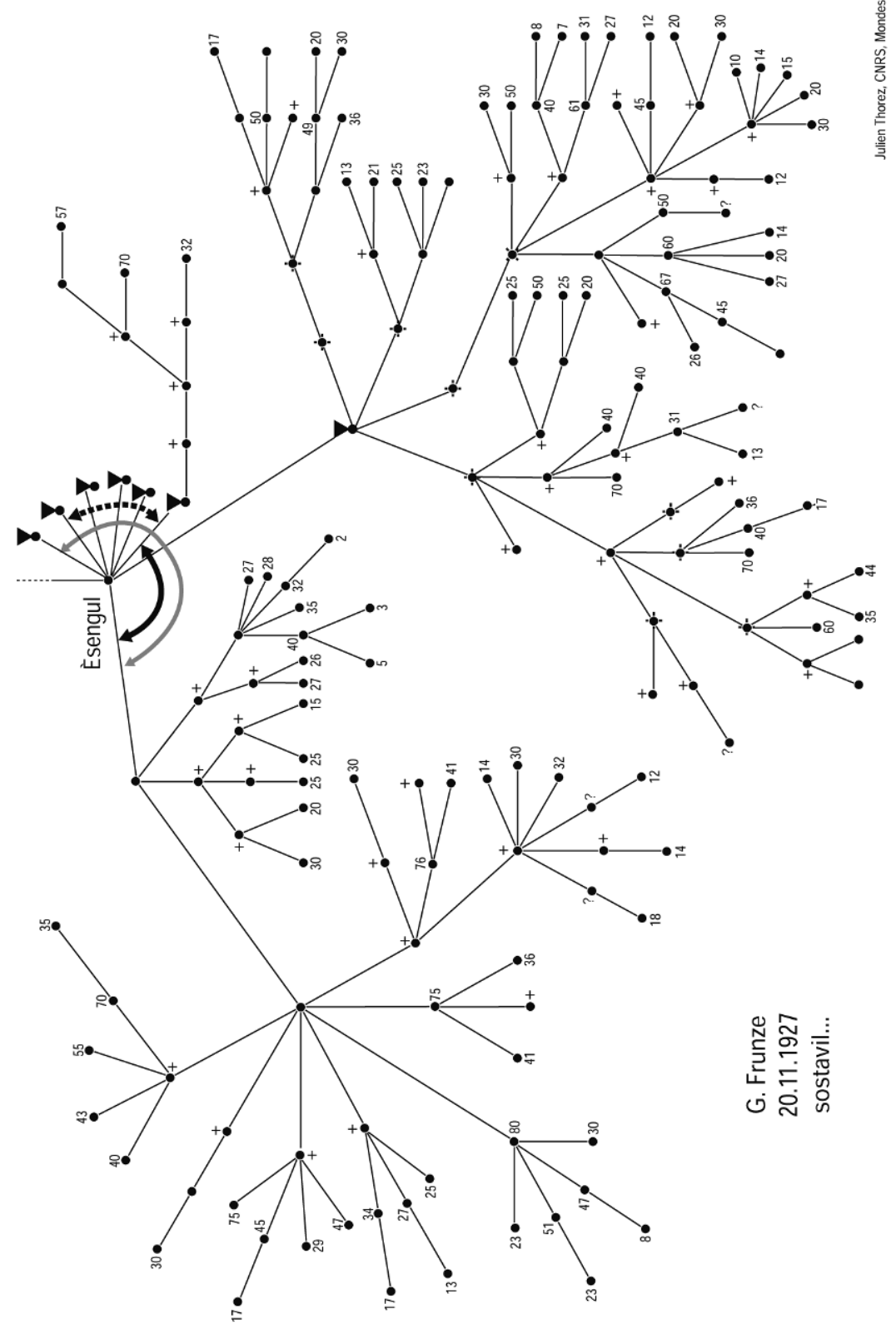

(๑) 